\title{
Probing Grand Unification Through Neutrino Oscillations, Leptogenesis, and Proton Decay*
}

\author{
Jogesh C. Pati \\ Department of Physics, University of Maryland, \\ College Park MD 20742
}

August 29, 2018

\begin{abstract}
Evidence in favor of supersymmetric grand unification including that based on the observed family multiplet-structure, gauge coupling unification, neutrino oscillations, baryogenesis, and certain intriguing features of quark-lepton masses and mixings is noted. It is argued that attempts to understand (a) the tiny neutrino masses (especially $\left.\Delta m^{2}\left(\nu_{2}-\nu_{3}\right)\right)$, (b) the baryon asymmetry of the universe (which seems to need leptogenesis), and (c) the observed features of fermion masses such as the ratio $m_{b} / m_{\tau}$, the smallness of $V_{c b}$ and the maximality of $\Theta_{\nu_{\mu} \nu_{\tau}}^{o s c}$, seem to select out the route to higher unification based on an effective string-unified $G(224)=S U(2)_{L} \times S U(2)_{R} \times S U(4)^{c}$ or $S O(10)$-symmetry, operative in $4 \mathrm{D}$, as opposed to other alternatives.

A predictive framework based on an effective $S O(10)$ or $G(224)$ symmetry possessing supersymmetry is presented that successfully describes the masses and mixings of all fermions including neutrinos. It also accounts for the observed baryon asymmetry of the universe by utilizing the process of leptogenesis, which is natural to this framework. It is argued that a conservative upper limit on the proton lifetime within this $S O(10) / G(224)$-framework, which is so far most successful, is given by $\left(\frac{1}{3}-2\right) \times 10^{34}$ years. This in turn strongly suggests that an improvement in the current sensitivity by a factor of five to ten (compared to SuperK) ought to reveal proton decay. Implications of this prediction for the next-generation nucleon decay and neutrino-detector are noted.
\end{abstract}

*Invited talks presented at the Erice School (September 2002), Neutrino Conference (Stony Brook, October 2002) and PASCOS Conference (Mumbai, January 2003), to appear in the respective Proceedings 


\section{Introduction And An Overview}

Since the discoveries (confirmations) of the atmospheric [1] and solar neutrino oscillations [2, 3], the neutrinos have emerged as being among the most effective probes into the nature of higher unification. Although almost the feeblest of all the entities of nature, simply by virtue of their tiny masses, they seem to possess a subtle clue to some of the deepest laws of nature pertaining to the unification-scale as well as the nature of the unification-symmetry. In this sense, the neutrinos provide us with a rare window to view physics at truly short distances. As we will see, these turn out to be as short as about $10^{-30} \mathrm{~cm}$. Furthermore, it appears most likely that the origin of their tiny masses may be at the root of the origin of matter-antimatter asymmetry in the early universe. In short, the neutrinos may well be crucial to our own origin!

The main purpose of my talk here today will be to present the intimate links that exist in the context of supersymmetric grand unification between the following phenomena: (i) neutrino oscillations, (ii) the masses and mixing of quarks and charged leptons, (iii) gauge coupling unification, (iv) baryogenesis via leptogenesis, and last but not least (v) proton decay.

To set the background for a discussion along these lines, let us first recall that with only left-handed neutrinos, the standard model based on the gauge symmetry $S U(2)_{L} \times U(1)_{Y} \times$ $S U(3)^{c}$, despite its numerous successes, fails to account for the magnitude of the massdifference square $\Delta m^{2}\left(\nu_{2}-\nu_{3}\right) \sim(1 / 20 \mathrm{eV})^{2}$ observed at Superkamiokande [1]. Incorporating effects of quantum gravity, ${ }^{1}$ the standard model can lead to a neutrino-mass $\sim 10^{-5} \mathrm{eV}$, which is, however, too small to account for the SuperK effect. One can in fact argue that, to understand the magnitude of the SuperK effect in any natural way, one would need new physics beyond the standard model at an effective mass-scale $\sim 10^{15} \mathrm{GeV}$, rather than at the Planck scale $\sim 10^{19} \mathrm{GeV}$ [4. Interestingly enough, one can link this effective mass-scale to the scale of meeting of the three gauge couplings (to be discussed below) which is around $2 \times 10^{16} \mathrm{GeV}$. That, in turn, hints at a link between the physics of neutrino-oscillations and grand unification!

The idea of "grand unification" was introduced in the early 1970's [5, 6, 7], purely on aesthetic grounds, in order to remove certain conceptual shortcomings of the standard model. Over the years, a set of key observations - some old and some new - have come to light, which together provide strong evidence in favor of this idea. Some of the observations in fact support the idea of both grand unification and low-energy supersymmetry [8, 9]. The evidence includes:

1. The observed family multiplet-structure - in particular the fact that the five (apparently disconnected) multiplets of the SM belonging to a family neatly become parts of a whole - a single multiplet - under grand unification, with all their quantum numbers predicted precisely as observed.

\footnotetext{
${ }^{1}$ See, e.g., S. Weinberg, Phys. Rev. Lett. 43, 1566 (1979); Proc. XXVI Int'l Conf. on High Energy Physics, Dallas, TX, 1992; E. Akhmedov, Z. Berezhiani and G. Senjanovic, Phys. Rev. D47, 3245 (1993). Assuming that quantum gravity could induce violation of lepton number, one may allow for an effective non-renormalizable operator of the form $\lambda_{L} L L H H / M_{p l}+$ h.c., scaled by $M_{p l}=1.2 \times 10^{19} \mathrm{GeV}$ with $\langle H\rangle \approx$ $250 \mathrm{GeV}$. Such an operator would, however, yield a rather small Majorana mass $m\left(\nu_{L}\right) \sim 10^{-5} \mathrm{eV}$. for the left-handed neutrinos, even for a maximal $\lambda_{L} \sim 1$, as mentioned in the text.
} 
2. The observed quantization of electric charge and the fact that the electron and the proton have exactly equal but opposite charges.

3. The dramatic meeting of the three gauge couplings that is found to occur at a scale $M_{X} \approx 2 \times 10^{16} \mathrm{GeV}$, when they are extrapolated from their values measured at LEP to higher energies, in the context of supersymmetry [10].

4. The tiny neutrino masses of the sort suggested by the discoveries/confirmations of atmospheric and solar neutrino oscillations. These, as we will see, not only go well with the scale of unification $M_{X}$ mentioned above but also help select out a class of unification-symmetries which provide the right-handed neutrinos $\left(\nu_{R}^{\prime} s\right)$ as a compelling feature and B-L as a local symmetry.

5. Certain intriguing features of the masses and mixings of the quarks and leptons, including the relation $m_{b}\left(M_{X}\right) \approx m_{\tau}$ and the largeness of the $\nu_{\mu}-\nu_{\tau}$ oscillation angle $\left(\sin ^{2} 2 \theta_{\nu_{\mu} \nu_{\tau}}^{\text {osc }} \geq 0.92\right)$ together with the smallness of $V_{c b}(\approx 0.04)[11]$.

6. And last but not least, the likely need for leptogenesis [12, 13, to account for the observed baryon-asymmetry of the universe, which seems to require once again the existence of superheavy right-handed neutrinos $\left(\nu_{R}^{\prime} s\right)$ and B-L as a local symmetry.

All of these features including the tiny neutrino masses and the observed baryon-asymmetry can be understood simply, and even quantitatively, within the concept of supersymmetric grand unification based on an effective symmetry in four dimensions, that is either

$$
\begin{gathered}
G(224)=S U(2)_{L} \times S U(2)_{R} \times S U(4)^{C}[5] \\
\text { or } \quad S O(10)[14 .
\end{gathered}
$$

Believing in a unified theory of all forces including gravity, it is of course attractive to presume that such an effective symmetry in $4 \mathrm{D}(G(224)$ or $S O(10))$ has its origin from a string theory [15] or the M-theory [16]. I will comment in Sec. 2 that, in the context of a string theory with the string-scale being close to the GUT-scale, the observed coupling unification may be understood even if the effective symmetry in $4 \mathrm{D}$, below the string scale, is non-simple like $G(224)$. A string-derived $G(224)$-solution may, however, have an advantage over an $S O(10)$-solution in that it can neatly avoid the so-called doublet-triplet splitting problem (generic to SUSY GUTs, see Sec. 2). Motivated by the desire to avoid this problem, there have in fact been several attempts in the literature (many rather recent) which successfully obtain semi-realistic $G(224)$-solutions in $4 \mathrm{D}$ from compactification of a string theory [17], or of an effective five or six dimensional GUT-theory [18]. For most purposes, in particular for considerations of fermion masses, neutrino-oscillations, and leptogenesis, the symmetries $G(224)$ and $S O(10)$ provide essentially the same advantages. Differences between them in considerations of proton decay will be noted in Sec. 5 .

Let us first recall the new features (relative to the SM) which are introduced through the symmetry $G(224)[5]$. Subject to left-right discrete symmetry $(L \leftrightarrow R)$, which is natural to $G(224)$, all members of the electron family become parts of a single left-right self-conjugate multiplet, consisting of:

$$
F_{L, R}^{e}=\left[\begin{array}{cccc}
u_{r} & u_{y} & u_{b} & \nu_{e} \\
d_{r} & d_{y} & d_{b} & e^{-}
\end{array}\right]_{L, R}
$$


The multiplets $F_{L}^{e}$ and $F_{R}^{e}$ are left-right conjugates of each other and transform respectively as $(2,1,4)$ and $(1,2,4)$ of $G(224)$; likewise for the muon and the tau families. The symmetry $S U(2)_{L}$ treats each column of $F_{L}^{e}$ as a doublet; likewise $S U(2)_{R}$ for $F_{R}^{e}$. The symmetry $S U(4)$-color unifies quarks and lepotons by treating each row of $F_{L}^{e}$ and $F_{R}^{e}$ as a quartet; thus lepton number is treated as the fourth color. As mentioned above, because of the parallelism between $S U(2)_{L}$ and $S U(2)_{R}$, the symmetry $G(224)$ naturally permits the notion that the fundamental laws of nature possess a left $\leftrightarrow$ right discrete symmetry (i.e. parity invariance) that interchanges $F_{L}^{e} \leftrightarrow F_{R}^{e}$ and $W_{L} \leftrightarrow W_{R}$. With suitable requirements on the Higgs sector, observed parity violation can be attributed, in this case, entirely to a spontaneous breaking of the $L \leftrightarrow R$ discrete symmetry [19].

Furthermore, the symmetry $G(224)$ introduces an elegant charge formula: $Q_{e m}=I_{3 L}+$ $I_{3 R}+(B-L) / 2$, that applies to all forms of matter (including quarks and leptons of all six flavors, Higgs and gauge bosons). Note that the weak hypercharge of the standard model, given by $Y_{W}=I_{3 R}+(B-L) / 2$, is now completely determined for all members of a family. Quite clearly, the charges $I_{3 L}, I_{3 R}$, and B-L, being generators respectively of $S U(2)_{L} S U(2)_{R}$, and $S U(4)^{c}$, are quantized; so also then is the electric charge $Q_{e m}$. Using the expression for $Q_{e m}$, one can now explain why the electron and the proton have exactly equal but opposite charges.

Note also that postulating either $S U(4)$-color or $S U(2)_{R}$ forces one to introduce a righthanded neutrino $\left(\nu_{R}\right)$ for each family as a singlet of the SM symmetry. This requires that there be sixteen two-component fermions in each family, as opposed to fifteen for the SM. Furthermore, $S U(4)$-color possesses B-L as one of its generators. This in turn helps to protect the Majorana masses of the right-handed neutrinos from being of the order string or Planck-scale. ${ }^{2}$ In addition, $S U(4)$-color provides the Dirac mass of the tau-neutrino by relating it to the top-quark mass at the unification-scale, and simultaneously the mass of the bottom quark in terms of that of the tau-lepton. In short, $S U(4)$-color introduces three characteristic features - i.e.

1. the right-handed neutrinos as a compelling feature,

2. B-L as a local symmetry, and

3. the two GUT-scale mass relations:

$$
m_{b}\left(M_{X}\right) \approx m_{\tau} \text { and } m\left(\nu_{\text {Dirac }}^{\tau}\right) \approx m_{\text {top }}\left(M_{X}\right)
$$

These two relations arise from the $\mathrm{SU}(4)$-color preserving leading entries in the fermion mass-matrices (see Sec. 3) which contribute to the masses of the third family. The subleading corrections to the fermion mass matrices that arise from $S U(4)$-color-breaking in the (B-L)-direction turn out to be important for the masses and mixings of the fermions belonging to the first two families [11. As we will see, these three ingredients, as well as the SUSY unification-scale $M_{X}$, play crucial roles in providing us with an understanding of the

\footnotetext{
${ }^{2}$ Without a protection by a local symmetry, $\nu_{R}^{\prime} s$ (being singlets of the SM) are likely to acquire Majorana masses of the order string or Planck scale through effects of quantum gravity. Such ultraheavy $\nu_{R}$-masses would, however, lead via the seesaw mechanism (see later), to too small masses for the light neutrinos $\left(\leq 10^{-5} \mathrm{eV}\right)$ and thereby to too small a value for $\Delta m_{23}^{2}$ compared to observation. Hence the need for B-L as an effective local symmetry in $4 \mathrm{D}$ near the string scale.
} 
tiny masses of the neutrinos as well as of the baryon-asymmetry of the universe, by utilizing respectively the seesaw mechanism [20] and the idea of leptogenesis [12. The success of the predictions in this regard (see below), speaks in favor of the seesaw mechanism and suggests that the effective symmetry in $4 \mathrm{D}$, below the string-scale, should contain SU(4)-color.

Now the minimal symmetry containing $S U(4)$-color on the one hand and also possessing a rationale for the quantization of electric charge on the other hand is provided by the group $G(224)$. The group $G(224)$ being isomorphic to $S O(4) \times S O(6)$ embeds nicely into the simple group $S O(10)$. The group $S O(10)$, which historically was proposed after the suggestion of $\mathrm{G}(224)$, of course retains all the advantages of $G(224)$, in particular the features (a)-(c) listed above. The interesting point is that $S O(10)$ even preserves the 16-plet multiplet structure of $G(224)$ by putting $\left\{F_{L}+\left(F_{R}\right)^{c}\right\}$ as its spinorial 16-dimensional representation, thereby avoiding the need for any new matter fermions. By contrast, if one extends $G(224)$ to the still higher symmetry $E_{6}$ [21], one must extend the family-structure from a 16 to a 27-plet, by postulating additional fermions.

Now utilizing the three ingredients (1), (2), and (3) listed above (thus assuming that $S U(4)$-color holds in 4D near the GUT-scale), together with the SUSY unification-scale $\left(M_{X}\right)$ and the seesaw mechanism, one arrives at a set of predictions (see Sec. 3) which include [1]:

$$
\begin{aligned}
m_{b}\left(m_{b}\right) & \approx 4.7-4.9 \mathrm{GeV} \\
m\left(\nu_{3}\right) & \approx\left(\frac{1}{24} \mathrm{eV}\right)\left(\frac{1}{2}-2\right) \\
\sin ^{2} 2 \theta_{\nu_{\mu} \nu_{\tau}}^{\mathrm{osc}} & \approx 0.99 \\
V_{c b} & \approx 0.044
\end{aligned}
$$

Each of these predictions agrees remarkably well with observations. The most intriguing feature is that this framework provides a compelling reason for why $V_{c b}$ is so small $(\approx 0.04)$, and simultaneously why $\sin ^{2} 2 \theta_{\nu_{\mu} \nu_{\tau}}$ is so large $(\approx 1)$, both in accord with observations. It is worth noting that the last two results, showing a sharp difference between $V_{c b}$ and $\theta_{\nu_{\mu} \nu_{\tau}}$, go against the often expressed (naive) view that the quark-lepton unification should lead to similar mixing angles in the quark and lepton sectors. Quite to the contrary, as we will see in Sec. 3, the minimal Higgs system provides a natural breaking of $S U(4)$-color along the (B-L)-direction which particularly contributes to a mixing between the second and the third families [11. That in turn provides a compelling group-theoretical reason for a distinction between the masses and mixings of the quarks and leptons as in fact observed empirically.

One important consequence of having an effective $G(224)$ or $S O(10)$-symmetry in $4 \mathrm{D}$ is that spontaneous breaking of such a symmetry (thereby of B-L) into the SM symmetry naturally generates Majorana masses of the RH neutrinos that are of order GUT-scale or smaller. In correlation with the flavor symmetries which provide the hierarchical masses of the quarks and the leptons, the Majorana masses of the three RH neutrinos are found to be 22]: $\left(M_{N_{1}}, M_{N_{2}}, M_{N_{3}}\right) \approx\left(10^{15}, 2 \times 10^{12},(1 / 3-2) \times 10^{10}\right) \mathrm{GeV}$. Given lepton number (thus of $\mathrm{B}-\mathrm{L}$ ) violation associated with these Majorana masses, and $\mathrm{C}$ and $\mathrm{CP}$ violating phases that generically arise in the Dirac and/or Majorana mass-matrices, the out of equilibrium decays of the lightest of these heavy RH neutrinos (produced after inflation ${ }^{3}$ ) into $l+H$ and $\bar{l}+\bar{H}$, and

\footnotetext{
${ }^{3}$ The lightest $N_{1}$ may be produced either thermally after reheating or non-thermally through inflaton-
} 
the corresponding SUSY modes, generates a lepton-asymmetry. The latter is then converted into a baryon-asymmetry by the electroweak sphaleron process [12, 13. In conjunction with an understanding of the fermion-masses and neutrino-oscillations (atmospheric and solar), the baryon excess thus generated is found to be (see Sec. 4 and Ref. [22]:

$$
Y_{B}=\left(\frac{n_{B}-n_{\bar{B}}}{n_{s}}\right) \approx\left(\sin 2 \phi_{21}\right)(7-100) \times 10^{-11}
$$

While the relevant phase angle $\phi_{21}$ arising from $\mathrm{C}$ and $\mathrm{CP}$-violating phases in the Dirac and Majorana mass-matrices of the neutrinos is not predictable within the framework, it is rather impressive that for plausible and natural values of the phase angle $\phi_{21} \approx \frac{1}{2}-\frac{1}{20}$ (say), the calculated baryon excess $Y_{B}$ agrees with the observed value based on big bang nucleosynthesis [23] and CMB data 24]. This may be contrasted from many alternative mechanisms, such as GUT and electroweak baryogenesis, which are either completely ineffective (owing to inflation and gravitino contraint) or yield too small a baryon excess even for a maximal phase. For a recent review and other relevant references on the topic of baryogenesis, see Ref. [25].

It should be stressed that the five predictions shown in Eqs(3) and (4), together make a crucial use of the three features (a)-(c) listed in Eq. (2), as well as of the SUSY unificationscale $M_{X}$ and the seesaw mechanism. Now the properties (a)-(c) are the distinguishing features of the symmetry $G(224)$. They are of course available within any symmetry that contains $G(224)$ as a subgroup. Thus they are present in $S O(10)$ and $E_{6}$, though not in $S U(5)$. Effective symmetries like $[S U(3)]^{3}\left[26\right.$ or $S U(2)_{L} \times S U(2)_{R} \times U(1)_{B-L} \times S U(3)^{c}$ 27] possess the first two features (a) and (b) but not (c). Flipped $S U(5) \times U(1)$ [28] on the other hand offers (a) and (b) but not the relation $m_{b}\left(M_{X}\right) \approx m_{\tau}$, which, however, is favored empirically.

The empirical success of the features (1)-(6), including specifically the predictions listed in Eqs. (3) and (41), seems to be non-trivial. Together they make a strong case for both the conventional ideas of supersymmetric grand unification ${ }^{4}$ and simultaneously for the symmetry $G(224)$ or $S O(10)$ being relevant to nature in four dimensions, just below the string scale.

As mentioned before, the main purpose of my talk here will be to present the intimate links that exist, in the context of supersymmetric grand unification based on an effective $G(224)$ or $S O(10)$ symmetry, between (i) neutrino oscillations, (ii) the masses and mixings of quarks and charged leptons, (iii) gauge coupling unification, (iv) baryogenesis via leptogenesis, and last but not least (v) proton decay.

Perhaps the most dramatic prediction of grand unification is proton decay. This important process which would provide the window to view physics at truly short distance $\left(<10^{-30}\right.$ $\mathrm{cm}$ ) and would greatly complement studies of neutrino oscillations in this regard is yet to be seen. One can, however, argue that the evidence listed above in favor of supersymmetric grand unification, based on an effective $G(224)$ or $S O(10)$ symmetry in $4 \mathrm{D}$, strongly suggests

decay during reheating. Both possibilities are considered in Sec. 3.

${ }^{4} \mathrm{By}$ "conventional" I mean gauge coupling unification occurring in $4 \mathrm{D}$ at a scale of few $\times 10^{16} \mathrm{Gev}$ (for the case of MSSM), with the string-scale being somewhat larger. This is to be contrasted from the case of large extra dimensions with unification occurring at the TeV-scale, on the one hand, or from 5D GUTs possessing unification only in higher dimensions leading to the SM symmetry $G(213)$ in $4 \mathrm{D}$, on the other hand. 
that an upper limit on proton lifetime is given by

$$
\tau_{\text {proton }} \lesssim\left(\frac{1}{3}-2\right) \times 10^{34} \mathrm{yrs}
$$

with $\bar{\nu} K^{+}$being the dominant mode, and quite possibly $\mu^{+} K^{o}$ and $e^{+} \pi^{o}$ being prominent. This in turn suggests that an improvement in the current sensitivity by a factor of five to ten (relative to SuperK) ought to reveal proton decay. A next-generation megaton-size detector of the kind being contemplated by the UNO [29] and the Hyperkamiokande [30] proposals would thus be needed to probe efficiently into the prediction of the supersymmetric $G(224) / S O(10)$-framework as regards proton decay.

I have discussed in a recent review 31 in some detail the updated results for proton decay in the context of supersymmetric $S U(5), S O(10)$ and $G(224)$-symmetries by taking into account (a) the recently improved (and enhanced) matrix elements as well as short and long-distance renormalization effects, (b) the dependence of the "standard" $\mathrm{d}=5$ protondecay operator on GUT-scale threshold corrections that are restricted by the requirement of natural coupling unification, and (c) its link with the masses and the mixings of all fermions including neutrinos [11. The latter give rise to a new set of $d=5$ operators, related to the Majorana masses of the RH neutrinos [32, which are found to be important. I will present a summary of the main results in this regard in Sec. 5 and also comment on the recent works which tend to avoid the standard $d=5$ proton decay operators which generically arise in the context of supersymmetric grand unification.

In Sec. 2, I discuss the implications of the meeting of the three gauge couplings in the context of string-unification. In Sec. 3, I discuss fermion masses and neutrino oscillations within a predictive framework based on the $G(224)$ or $S O(10)$ symmetry in $4 \mathrm{D}$ which lead to predictions of the type shown in Eq.(33), and in Sec. 4, I discuss leptogenesis within the same framework. Results on proton decay which arise within this framework and within related approaches are summarized in Sec. 5. Concluding remarks are presented in Sec. 6, where the case for building a major underground detector with improved sensitivity to detecting proton decay and neutrino oscillations is made.

\section{MSSM Versus String Unifications: $G(224)$ Versus $S O(10)$ as Effective Symmetries}

As mentioned in the introduction, the three gauge couplings are found to meet when they are extrapolated from their values measured at LEP to higher energies by assuming that the SM is replaced by the minimal supersymmetric standard model (MSSM) above a threshold of about $1 \mathrm{TeV}$ [10]. The meeting occurs to a very good approximation, barring a few percent discrepancy which can legitimately be attributed to GUT-scale threshold corrections. Their scale of meeting is given by:

$$
M_{X} \approx 2 \times 10^{16} \mathrm{GeV}(\operatorname{MSSM} \text { or SUSY } S U(5))
$$

This dramatic meeting of the three gauge couplings provides a strong support for the ideas of both grand unification and supersymmetry, as being relevant to physics at short distances $\lesssim\left(10^{16} \mathrm{GeV}\right)^{-1}$. 
In addition to being needed for achieving coupling unification there is of course an independent motivation for low-energy supersymmetry - i.e. for the existence of SUSY partners of the standard model particles with masses of order $1 \mathrm{TeV}$. This is because it protects the Higgs boson mass from getting large quantum corrections, which would (otherwise) arise from grand unification and Planck scale physics. It thereby provides at least a technical resolution of the so-called gauge-hierarchy problem. In this sense low-energy supersymmetry seems to be needed for the consistency of the hypothesis of grand unification. Supersymmetry is of course also needed for the consistency of string theory. It is fortunate that low-energy supersymmetry can be tested at the LHC, and possibly at the Tevatron, and the proposed NLC.

The most straightforward interpretation of the observed meeting of the three gauge couplings and of the scale $M_{X}$, is that a supersymmetric grand unification symmetry (often called GUT symmetry), like $S U(5)$ or $S O(10)$, breaks spontaneously at $M_{X}$ into the standard model symmetry $G(213)$, and that supersymmetry-breaking induces soft masses of order one $\mathrm{TeV}$.

Even if supersymmetric grand unification may well be a good effective theory below a certain scale $M \gtrsim M_{X}$, it ought to have its origin within an underlying theory like the string/M theory. Such a theory is needed to unify all the forces of nature including gravity, and to provide a good quantum theory of gravity. It is also needed to provide a rationale for the existence of flavor symmetries (not available within grand unification), which distinguish between the three families and can resolve certain naturalness problems including those associated with inter-family mass hierarchy. As alluded to in the introduction, in the context of string or M-theory, an alternative interpretation of the observed meeting of the gauge couplings is however possible. This is because, even if the effective symmetry in four dimensions emerging from a higher dimensional string theory is non-simple, like $G(224)$ or even $G(213)$, string theory can still ensure familiar unification of the gauge couplings at the string scale. In this case, however, one needs to account for the small mismatch between the MSSM unification scale $M_{X}$ (given above), and the string unification scale, given by $M_{s t} \approx g_{s t} \times 5.2 \times 10^{17} \mathrm{GeV} \approx 3.6 \times 10^{17} \mathrm{GeV}$ (Here we have put $\alpha_{s t}=\alpha_{\mathrm{GUT}}(\mathrm{MSSM}) \approx 0.04$ ) 33. Possible resolutions of this mismatch have been proposed. These include: (i) utilizing the idea of string-duality [34] which allows a lowering of $M_{s t}$ compared to the value shown above, or alternatively (ii) the idea of the so-called "Extended Supersymmetric Standard Model" (ESSM) that assumes the existence of two vector-like families, transforming as $(\mathbf{1 6}+\overline{\mathbf{1 6}})$ of $S O(10)$, with masses of order one $\mathrm{TeV}$ [35], in addition to the three chiral families. The latter leads to a semi-perturbative unification by raising $\alpha_{\mathrm{GUT}}$ to about 0.25-0.3. Simultaneously, it raises $M_{X}$, in two loop, to about $(1 / 2-2) \times 10^{17} \mathrm{GeV}$. (Other mechanisms resolving the mismatch are reviewed in Ref. [36]). In practice, a combination of the two mechanisms mentioned above may well be relevant. ${ }^{5}$

\footnotetext{
${ }^{5}$ I have in mind the possibility of string-duality 34 lowering $M_{s t}$ for the case of semi-perturbative unification in ESSM (for which $\alpha_{s t} \approx 0.25$, and thus, without the use of string-duality, $M_{s t}$ would have been about $10^{18} \mathrm{GeV}$ ) to a value of about $(1-2) \times 10^{17} \mathrm{GeV}$ (say), and semi-perturbative unification [35] raising the MSSM value of $M_{X}$ to about $5 \times 10^{16} \mathrm{GeV} \approx M_{s t}$ (1/2 to $\left.1 / 4\right)$ (say). In this case, an intermediate symmetry like $G(224)$ emerging at $M_{s t}$ would be effective only within the short gap between $M_{s t}$ and $M_{X}$, where it would break into $G(213)$. Despite this short gap, one would still have the benefits of $S U(4)$-color that are needed to understand neutrino masses (see Section 3), and to implement baryogenesis via leptogenesis. At the same time, since the gap is so small, the couplings of $G(224)$, unified at $M_{s t}$ would remain essentially so
} 
While the mismatch can thus quite plausibly be removed for a non-GUT string-derived symmetry like $G(224)$ or $G(213)$, a GUT symmetry like $S U(5)$ or $S O(10)$ would have an advantage in this regard because it would keep the gauge couplings together between $M_{s t}$ and $M_{X}$ (even if $M_{X} \sim M_{s t} / 20$ ), and thus not even encounter the problem of a mismatch between the two scales. A supersymmetric four dimensional GUT-solution [like $S U(5)$ or $S O(10)$ ], however, has a possible disadvantage as well, because it needs certain color triplets to become superheavy by the so-called doublet-triplet splitting mechanism in order to avoid the problem of rapid proton decay. However, no such mechanism has emerged yet, in string theory, for the four-dimensional GUT-like solutions 37. ${ }^{6}$

Non-GUT string solutions, based on symmetries like $G(224)$ or $G(2113)$ for example, have a distinct advantage in this regard, in that the dangerous color triplets, which would induce rapid proton decay, are often naturally projected out for such solutions [15, 17, 38. Furthermore, the non-GUT solutions invariably possess new "flavor" gauge symmetries, which distinguish between families and also among members within a family. These symmetries are immensely helpful in explaining qualitatively the observed fermion mass-hierarchy (see e.g. Ref. [38]) and resolving the so-called naturalness problems of supersymmetry such as those pertaining to the issues of squark-degeneracy [39, CP violation [40] and quantum gravity-induced rapid proton decay [41].

Weighing the advantages and possible disadvantages of both, it seems hard at present to make a priori a clear choice between a GUT versus a non-GUT string-solution. As expressed elsewhere 42, it therefore seems prudent to keep both options open and pursue their phenomenological consequences. Given the advantages of $G(224)$ or $S O(10)$ in understanding the neutrino masses and implementing leptogenesis (see Sections 3 and 4), I will thus proceed by assuming that either a suitable four dimensional $G(224)$-solution with the scale $M_{X}$ being close to $M_{s t}$ (see footnote 5 ), or a realistic four-dimensional $S O(10)$-solution with the desired mechanism for doublet-triplet splitting, emerges effectively from an underlying string theory, at the "conventional" string-scale $M_{s t} \sim 10^{17}-10^{18} \mathrm{GeV}$, and that the $G(224) / S O(10)$ symmetry in turn breaks spontaneously at the conventional GUT-scale of $M_{X} \sim 2 \times 10^{16} \mathrm{GeV}$ ( or at $M_{X} \sim 5 \times 10^{16} \mathrm{GeV}$ for the case of ESSM, as discussed in footnote $4)$ to the standard model symmetry $G(213)$. The extra dimensions of string/M-theory are assumed to be tiny with sizes $\leq M_{X}^{-1} \sim 10^{-30} \mathrm{~cm}$, so as not to disturb the successes of GUT. In short, I assume that essentially the conventional (good old) picture of grand unification, proposed and developed sometime ago [5, 6, [7, 10], holds as a good effective theory above the unification scale $M_{X}$ and up to some high scale $M \lesssim M_{\text {st }}$, with the added presumption that it may have its origin from the string/M-theory. ${ }^{7}$

We will see that with the broad assumption mentioned above, an economical and predictive framework emerges, which successfully accounts for a host of observed phenomena pertaining to the masses and the mixings of all fermions, including neutrinos, and the baryon

at $M_{X}$, so as to match with the "observed" coupling unification, of the type suggested in Ref. [35.

${ }^{6}$ Some alternative mechanisms for doublet-triplet splitting, and for suppression of the $d=5$ proton decay operators have been proposed in the context of higher dimensional theories. These will be mentioned briefly in Section 5.

${ }^{7}$ Alternative scenarios such as those based on TeV-scale large extra dimensions [4], or string-scale being at a few $\mathrm{TeV}$ [4], or submillimeter-size even larger extra dimensions with the fundamental scale of quantum gravity being a few $\mathrm{TeV}$ 45, though intriguing, do not seem to provide simple explanations of these features: (a), (b), and (c). They will be mentioned briefly in Section 5.2.5. 
asymmetry of the universe. It also makes some crucial testable predictions for proton decay.

\section{Link Between Fermion Masses and Neutrino Oscil- lations within a $G(224) / S O(10)$ Framework}

Following Ref. [11, I now present a simple and predictive fermion mass-matrix based on $S O(10)$ or the $G(224)$-symmetry. ${ }^{8}$ One can obtain such a mass mass-matrix for the fermions by utilizing only the minimal Higgs system that is needed to break the gauge symmetry $S O(10)$ to $S U(3)^{c} \times U(1)_{e m}$. It consists of the set:

$$
H_{\text {minimal }}=\left\{45_{\mathbf{H}}, 1 \mathbf{6}_{\mathbf{H}}, \overline{\mathbf{1 6}}_{\mathbf{H}}, 10_{\mathbf{H}}\right\}
$$

Of these, the VEV of $\left\langle\mathbf{4} \mathbf{5}_{\mathbf{H}}\right\rangle \sim M_{X}$ breaks $S O(10)$ in the B-L direction to $G(2213)=$ $S U(2)_{L} \times S U(2)_{R} \times U(1)_{B-L} \times S U(3)^{c}$, and those of $\left\langle\mathbf{1 6}_{\mathbf{H}}\right\rangle=\left\langle\overline{\mathbf{1 6}}_{\mathbf{H}}\right\rangle$ along $\left\langle\tilde{\bar{\nu}}_{R H}\right\rangle$ and $\left\langle\tilde{\nu}_{R H}\right\rangle$ break $G(2213)$ into the SM symmetry $G(213)$ at the unification-scale $M_{X}$. Now $G(213)$ breaks at the electroweak scale by the VEV of $\left\langle\mathbf{1 0}_{\mathbf{H}}\right\rangle$ to $S U(3)^{c} \times U(1)_{e m} .{ }^{9}$

The question is: can the minimal Higgs system provide a realistic pattern for fermion masses and mixings? Now $\mathbf{1 0}_{\mathbf{H}}$ (even several 10's) can not provide certain desirable features - i.e. family-antisymmetry and (B-L)-dependence in the mass matrices - which are, however, needed respectively to suppress $V_{c b}$ while enhancing $\theta_{\nu_{\mu} \nu_{\tau}}$ on the one hand, and accounting for features such as $m_{\mu}^{0} \neq m_{s}^{0}$ on the other hand (see e.g. Ref. [31, Sec. 5). Furthermore, a single $\mathbf{1 0} \mathbf{H}$ cannot generate CKM mixings. At the same time, $\mathbf{1 0}_{\mathbf{H}}$ is the only multiplet among the ones in the minimal Higgs system (Eq. (6) ) which can have cubic couplings with the matter fermions which are in the 16's. This impasse disappears as soon as one allows for not only cubic but also effective non-renormalizable quartic couplings of the minimal set of Higgs fields with the fermions. Such effective couplings can of course arise quite naturally through exchanges of superheavy states (e.g. those in the string-tower or those having GUT-scale masses) involving renormalizable couplings, and/or through quantum gravity.

The $3 \times 3$ Dirac masses matrices for the four sectors $(u, d, l, \nu)$ proposed in Ref. 11] are motivated in part by the group theory of $S O(10) / G(224)$, which severely restricts the effective cubic and quartic couplings (and thus the associated mass-patterns), for the minimal Higgs system. They are also motivated in part by the notion that flavor symmetries [47] distinguishing between the three families lead to a hierarchical pattern for the mass matrices

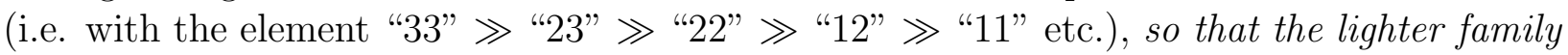
gets its mass primarily through its mixing with the heavier ones. It turns out that the allowed forms of effective couplings and the corresponding pattern of mass-matrices, satisfying the

\footnotetext{
${ }^{8}$ I will present the Higgs system for $S O(10)$. The discussion would remain essentially unaltered if one uses the corresponding $G(224)$-submultiplets instead.

${ }^{9}$ Large dimensional tensorial multiplets of $S O(10)$ like $\mathbf{1 2 6}_{\mathbf{H}}, \overline{\mathbf{1 2 6}}_{H}, \mathbf{1 2 0}_{\mathbf{H}}$, and $\mathbf{5 4}_{\mathbf{H}}$ are not used for the purpose in part because they do not seem to arise at least in weakly interacting heterotic string solutions [46, and in part because they tend to give too large threshold corrections to $\alpha_{3}\left(m_{Z}\right)$ (typically exceeding $20 \%$ ), which would render observed coupling unification fortuitous [see e.g. discussions in Appendix D of Ref. [11].
} 
constraints of group-theory and flavor-hierarchy (as above), are rather unique, barring a few discrete variants. The mass matrices proposed in Ref. [1] are as follows: ${ }^{10} 11$

$$
\begin{aligned}
& M_{u}=\left[\begin{array}{ccc}
0 & \epsilon^{\prime} & 0 \\
-\epsilon^{\prime} & 0 & \sigma+\epsilon \\
0 & \sigma-\epsilon & 1
\end{array}\right] \mathcal{M}_{u}^{0} ; \quad M_{d}=\left[\begin{array}{ccc}
0 & \eta^{\prime}+\epsilon^{\prime} & 0 \\
\eta^{\prime}-\epsilon^{\prime} & 0 & \eta+\epsilon \\
0 & \eta-\epsilon & 1
\end{array}\right] \mathcal{M}_{d}^{0} \\
& M_{\nu}^{D}=\left[\begin{array}{ccc}
0 & -3 \epsilon^{\prime} & 0 \\
-3 \epsilon^{\prime} & 0 & \sigma-3 \epsilon \\
0 & \sigma+3 \epsilon & 1
\end{array}\right] \mathcal{M}_{u}^{0} ; \quad M_{l}=\left[\begin{array}{ccc}
0 & \eta^{\prime}-3 \epsilon^{\prime} & 0 \\
\eta^{\prime}+3 \epsilon^{\prime} & 0 & \eta-3 \epsilon \\
0 & \eta+3 \epsilon & 1
\end{array}\right] \mathcal{M}_{d}^{0}
\end{aligned}
$$

These matrices are defined in the gauge basis and are multiplied by $\bar{\Psi}_{L}$ on left and $\Psi_{R}$ on right. For instance, the row and column indices of $M_{u}$ are given by $\left(\bar{u}_{L}, \bar{c}_{L}, \bar{t}_{L}\right)$ and $\left(u_{R}, c_{R}, t_{R}\right)$ respectively. Note the group-theoretic up-down and quark-lepton correlations: the same $\sigma$ occurs in $M_{u}$ and $M_{\nu}^{D}$, and the same $\eta$ occurs in $M_{d}$ and $M_{l}$. It will become clear that the $\epsilon$ and $\epsilon^{\prime}$ entries are proportional to B-L and are antisymmetric in the family space (as shown above). Thus, the same $\epsilon$ and $\epsilon^{\prime}$ occur in both $\left(M_{u}\right.$ and $\left.M_{d}\right)$ and also in $\left(M_{\nu}^{D}\right.$ and $M_{l}$ ), but $\epsilon \rightarrow-3 \epsilon$ and $\epsilon^{\prime} \rightarrow-3 \epsilon^{\prime}$ as $q \rightarrow l$. Such correlations result in an enormous reduction of parameters and thus in increased predictivity. Although the entries $\sigma, \eta, \epsilon, \eta^{\prime}$, and $\epsilon^{\prime}$ will be treated as parameters, consistent with assignment of flavor-symmetry charges (see below), we would expect them to be hierarchical with $(\sigma, \eta, \epsilon) \sim 1 / 10$ and $\left(\eta^{\prime}, \epsilon^{\prime}\right) \sim 10^{-3}-10^{-4}$ (say). Such a hierarchical pattern for the mass-matrices can be obtained, using a minimal Higgs system $\mathbf{4 5} \mathbf{5}_{H}, \mathbf{1 6}_{H}, \overline{\mathbf{1 6}}_{H}$ and $\mathbf{1 0}_{H}$ and a singlet $S$ of $S O(10)$, through effective couplings as follows 49:

$$
\begin{aligned}
\mathcal{L}_{\text {Yuk }} & =h_{33} \mathbf{1 6}_{3} \mathbf{1 6}_{3} \mathbf{1 0}_{H} \\
& +\left[h_{23} \mathbf{1 6}_{2} \mathbf{1 6}_{3} \mathbf{1 0}_{H}(S / M)+a_{23} \mathbf{1 6}_{2} \mathbf{1 6}_{3} \mathbf{1 0}_{H}\left(\mathbf{4 5}_{H} / M^{\prime}\right)(S / M)^{p}+g_{23} \mathbf{1 6}_{2} \mathbf{1 6}_{3} \mathbf{1 6}_{H}^{d}\left(\mathbf{1 6}_{H} / M^{\prime \prime}\right)(S / M)^{q}\right] \\
& +\left[h_{22} \mathbf{1 6}_{2} \mathbf{1 6}_{2} \mathbf{1 0}_{H}(S / M)^{2}+g_{22} \mathbf{1 6}_{2} \mathbf{1 6}_{2} \mathbf{1 6}_{H}^{d}\left(\mathbf{1 6}_{H} / M^{\prime \prime}\right)(S / M)^{q+1}\right] \\
& +\left[g_{12} \mathbf{1 6}_{1} \mathbf{1 6}_{2} \mathbf{1 6}_{H}^{d}\left(\mathbf{1 6}_{H} / M^{\prime \prime}\right)(S / M)^{q+2}+a_{12} \mathbf{1 6}_{1} \mathbf{1 6}_{2} \mathbf{1 0}_{H}\left(\mathbf{4 5}_{H} / M^{\prime}\right)(S / M)^{p+2}\right]
\end{aligned}
$$

Typically we expect $M^{\prime}, M^{\prime \prime}$ and $M$ to be of order $M_{\text {string }}$ [50]. The VEV's of $\left\langle\mathbf{4 5} \mathbf{5}_{H}\right\rangle$ (along B-L), $\left\langle\mathbf{1 6}_{H}\right\rangle=\left\langle\overline{\mathbf{1 6}}_{H}\right\rangle$ (along standard model singlet sneutrino-like component) and of the $\mathrm{SO}(10)$-singlet $\langle S\rangle$ are of the GUT-scale, while those of $\mathbf{1 0}_{H}$ and of the down type $\mathrm{SU}(2)_{L}$-doublet component in $\mathbf{1 6}_{H}$ (denoted by $\mathbf{1 6}_{H}^{d}$ ) are of the electroweak scale [11, 51. Depending upon whether $M^{\prime}\left(M^{\prime \prime}\right) \sim M_{\mathrm{GUT}}$ or $M_{\text {string }}$ (see comment in [50]), the exponent $p(q)$ is either one or zero 52 .

The entries 1 and $\sigma$ arise respectively from $h_{33}$ and $h_{23}$ couplings, while $\hat{\eta} \equiv \eta-\sigma$ and $\eta^{\prime}$ arise respectively from $g_{23}$ and $g_{12}$-couplings. The (B-L)-dependent antisymmetric entries $\epsilon$

\footnotetext{
${ }^{10}$ The zeros in "11", "13", "31", and " $22 "$ elements signify that they are relatively small. For instance, the " 22 "-elements are set to zero because (restricted by flavor symmetries, see below), they are meant to be

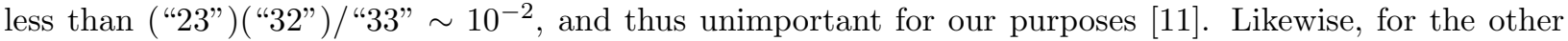
"zeros."

${ }^{11}$ A somewhat analogous pattern, also based on $S O(10)$, has been proposed by C. Albright and S. Barr [AB] 48. One major difference between the work of $\mathrm{AB}$ and that of BPW 11 is that the former introduces the so-called "lop-sided" pattern in which some of the "23" elements are even greater than the "33" element; in BPW on the otherhand, the pattern is consistently hierarchical with individual "23" elements (like $\eta, \epsilon$ and $\sigma$ ) being much smaller in magnitude than the "33" element of 1 .
} 
and $\epsilon^{\prime}$ arise respectively from the $a_{23}$ and $a_{12}$ couplings. This is because, with $\left\langle\mathbf{4} \mathbf{5}_{H}\right\rangle \propto \mathrm{B}-\mathrm{L}$, the product $\mathbf{1 0} \mathbf{0}_{H} \times \mathbf{4 5} \mathbf{5}_{H}$ contributes as a $\mathbf{1 2 0}$, whose coupling is family-antisymmetric. Thus, for the minimal Higgs system (see Eq. 6), (B-L)-dependence can enter only through family off-diagonal couplings of $\mathbf{1 0}_{\mathbf{H}} \cdot \mathbf{4 5}_{\mathbf{H}}$ as in $a_{23}$ and $a_{12}$-terms. Thus, for such a system, the diagonal "33" entries are necessarily (B-L)-independent (as shown in Eq. (7)). This in turn makes the relations like $m_{b}\left(M_{X}\right) \approx m_{\tau}$ (barring corrections of order $\epsilon^{2}$ [11]) robust. This feature would, however, be absent if one had used $\overline{\mathbf{1 2 6}}_{\mathbf{H}}$, whose coupling is family-symmetric and can give (B-L) dependent contributions to the "33"-elements.

As alluded to above, such a hierarchical form of the mass-matrices, with $h_{33}$-term being dominant, is attributed in part to flavor gauge symmetry(ies) that distinguishes between the three families [53, and in part to higher dimensional operators involving for example $\left\langle\mathbf{4 5} \mathbf{5}_{H}\right\rangle / M^{\prime}$ or $\left\langle\mathbf{1 6}_{H}\right\rangle / M^{\prime \prime}$, which are supressed by $M_{\mathrm{GUT}} / M_{\text {string }} \sim 1 / 10$, if $M^{\prime}$ and $/$ or $M^{\prime \prime} \sim$ $M_{\text {string. }}$.

To discuss the neutrino sector one must specify the Majorana mass-matrix of the RH neutrinos as well. These arise from the effective couplings of the form [54]:

$$
\mathcal{L}_{\mathrm{Maj}}=f_{i j} \mathbf{1 6}_{i} \mathbf{1 6}_{j} \overline{\mathbf{1 6}}_{H} \overline{\mathbf{1 6}}_{H} / M
$$

where the $f_{i j}$ 's include appropriate powers of $\langle S\rangle / M$, in accord with flavor charge assignments of $\mathbf{1 6}_{i}$ (see [53]). For the $f_{33}$-term to be leading, we must assign the charge $-a$ to $\overline{\mathbf{1 6}}_{H}$. This leads to a hierarchical form for the Majorana mass-matrix [1]:

$$
M_{R}^{\nu}=\left[\begin{array}{lll}
x & 0 & z \\
0 & 0 & y \\
z & y & 1
\end{array}\right] M_{R}
$$

Following the flavor-charge assignments given in [53, we expect $|y| \sim\langle S / M\rangle \sim 1 / 10,|z| \sim$ $(\langle S / M\rangle)^{2} \sim 10^{-2}(1$ to $1 / 2),|x| \sim(\langle S / M\rangle)^{4} \sim\left(10^{-4}-10^{-5}\right)$ (say). The "22" element (not shown $)$ is $\sim(\langle S / M\rangle)^{2}$ and its magnitude is taken to be $<\left|y^{2} / 3\right|$, while the "12" element (not shown) is $\sim(\langle S / M\rangle)^{3}$. We expect

$$
M_{R}=f_{33}\left\langle\overline{\mathbf{1 6}}_{\mathbf{H}}\right\rangle^{2} / M_{\text {string }} \approx 10^{15} \mathrm{GeV}(1 / 2-2)
$$

where we have put $\left\langle\overline{\mathbf{1 6}}_{\mathbf{H}}\right\rangle \approx M_{X} \approx 2 \times 10^{16} \mathrm{GeV}, M_{\text {string }} \approx 4 \times 10^{17} \mathrm{GeV}\left[33\right.$, and $f_{33} \approx 1$, and have allowed for an uncertainty by a factor of 2 in the estimate around a centrally expected value of about $10^{15} \mathrm{GeV}$. Allowing for $2-3$ family-mixing in the Dirac and the Majorana sectors as in Eqs. 7 and 10, the seesaw mechanism leads to [1]:

$$
m\left(\nu_{3}\right) \approx B \frac{m\left(\nu_{\text {Dirac }}^{\tau}\right)^{2}}{M_{R}}
$$

The quantity $B$ represents the effect of 2-3 mixing and is given by $B=(\sigma+3 \epsilon)(\sigma+3 \epsilon-2 y) / y^{2}$ (see Eq. (24) of Ref. [1]). Thus $B$ is fully calculable within the model once the parameters $\sigma, \eta, \epsilon$, and $y$ are determined in terms of inputs involving some quark and lepton masses (as noted below $)$. In this way, one obtains $B \approx(2.9 \pm 0.5)$. The Dirac mass of the tau-neutrino is obtained by using the $S U(4)$-color relation (see Eq. (2)): $m\left(\nu_{\text {Dirac }}^{\tau}\right) \approx m_{\text {top }}\left(M_{X}\right) \approx 120 \mathrm{GeV}$. One thus obtains from Eq. (12):

$$
m\left(\nu_{3}\right) \approx \frac{(2.9)(120 \mathrm{GeV})^{2}}{10^{15} \mathrm{GeV}}(1 / 2-2) \approx(1 / 24 \mathrm{eV})(1 / 2-2)
$$


Noting that for hierarchical entries - i.e. for $(\sigma, \epsilon$, and $y) \sim 1 / 10$ - one naturally obtains a hierarchical spectrum of neutrino-masses: $m\left(\nu_{1}\right) \lesssim m\left(\nu_{2}\right) \sim(1 / 10) m\left(\nu_{3}\right)$, we thus get:

$$
\left[\sqrt{\Delta m_{23}^{2}}\right]_{\text {Theory }} \approx m\left(\nu_{3}\right) \approx(1 / 24 \mathrm{eV})(1 / 2-2)
$$

This agrees remarkably well with the SuperK value of $\left(\sqrt{\Delta m_{23}^{2}}\right)_{\mathrm{SK}}(\approx 1 / 20 \mathrm{eV})$, which lies in the range of nearly $(1 / 15$ to $1 / 30) \mathrm{eV}$. As mentioned in the introduction, the success of this prediction provides clear support for (i) the existence of $\nu_{R}$, (ii) the notion of $S U(4)$-color symmetry that gives $m\left(\nu_{\text {Dirac }}^{\tau}\right)$, (iii) the SUSY unification-scale that gives $M_{R}$, and (iv) the seesaw mechanism.

We note that alternative symmetries such as $S U(5)$ would have no compelling reason to introduce the $\nu_{R}$ 's. Even if one did introduce $\nu_{R}^{i}$ by hand, there would be no symmetry to relate the Dirac mass of $\nu_{\tau}$ to the top quark mass. Thus $m\left(\nu_{\text {Dirac }}^{\tau}\right)$ would be an arbitrary parameter in $S U(5)$. Furthermore, without B-L as a local symmetry, the Majorana masses of the RH neutrinos, which are singlets of $S U(5)$, can naturally be of order string scale $\sim 4 \times 10^{17} \mathrm{GeV}$ (say). That would, however, give too small a mass for $m\left(\nu_{3}\right)\left(<10^{-4} \mathrm{eV}\right)$ compared to the SuperK value.

Other effective symmetries such as $\left.[S U(3)]^{3}\right]\left[26\right.$ and $S U(2)_{L} \times S U(2)_{R} \times U(1)_{B-L} \times$ $S U(3)^{C}$ [27] would give $\nu_{R}$ and B-L as a local symmetry, but not the desired $S U(4)$-color mass-relations: $m\left(\nu_{\text {Dirac }}^{\tau}\right) \approx m_{t}\left(M_{X}\right)$ and $m_{b}\left(M_{X}\right) \approx m_{\tau}$. Flip $S U(5) \times U(1)$ [28] on the other hand would yield the desired features for the neutrino-system, but not the $b-\tau$ mass relation. Thus, combined with the observed $b / \tau$ mass-ratio, the SuperK data on atmospheric neutrino oscillation seems to clearly select out the effective symmetry in $4 \mathrm{D}$ being either $G(224)$ or $S O(10)$, as opposed to the other alternatives mentioned above. It is in this sense that the neutrinos, by virtue of their tiny masses, provide crucial information on the unification-scale as well as on the nature of the unification-symmetry in $4 D$, as alluded to in the introduction.

Ignoring possible phases in the parameters and thus the source of CP violation for a moment, as was done in Ref. [11, the parameters $\left(\sigma, \eta, \epsilon, \epsilon^{\prime}, \eta^{\prime}, \mathcal{M}_{u}^{0}, \mathcal{M}_{D}^{0}\right.$, and $\left.y\right)$ can be determined by using, for example, $m_{t}^{\text {phys }}=174 \mathrm{GeV}, m_{c}\left(m_{c}\right)=1.37 \mathrm{GeV}, m_{S}(1 \mathrm{GeV})=110$ $116 \mathrm{MeV}, m_{u}(1 \mathrm{GeV})=6 \mathrm{MeV}$, the observed masses of $e, \mu$, and $\tau$ and $m\left(\nu_{2}\right) / m\left(\nu_{3}\right) \approx$ $1 /(7 \pm 1)$ (as suggested by a combination of atmospheric [1] and solar neutrino data [2], the latter corresponding to the LMA MSW solution, see below) as inputs. One is thus led, for this CP conserving case, to the following fit for the parameters, and the associated predictions [11. [In this fit, we leave the small quantities $x$ and $z$ in $M_{R}^{\nu}$ undetermined and proceed by assuming that they have the magnitudes suggested by flavor symmetries (i.e., $x \sim\left(10^{-4}-10^{-5}\right)$ and $z \sim 10^{-2}(1$ to $1 / 2$ ) (see remarks below Eq. (10) $)$ ]:

$$
\begin{gathered}
\sigma \approx 0.110, \quad \eta \approx 0.151, \quad \epsilon \approx-0.095, \quad\left|\eta^{\prime}\right| \approx 4.4 \times 10^{-3}, \\
\epsilon^{\prime} \approx 2 \times 10^{-4}, \quad \mathcal{M}_{u}^{0} \approx m_{t}\left(M_{X}\right) \approx 120 \mathrm{GeV}, \\
\mathcal{M}_{D}^{0} \approx m_{b}\left(M_{X}\right) \approx 1.5 \mathrm{GeV}, \quad y \approx-(1 / 17) .
\end{gathered}
$$


These in turn lead to the following predictions for the quarks and light neutrinos [11]:12

$$
\begin{aligned}
& m_{b}\left(m_{b}\right) \approx(4.7-4.9) \mathrm{GeV}, \\
& m\left(\nu_{3}\right) \approx(1 / 24 \mathrm{eV})(1 / 2-2) \text {, } \\
& \left.V_{c b} \approx\left|\sqrt{\frac{m_{s}}{m_{b}}}\right| \frac{\eta+\epsilon}{\eta-\epsilon}\right|^{1 / 2}-\sqrt{\frac{m_{c}}{m_{t}}}\left|\frac{\sigma+\epsilon}{\sigma-\epsilon}\right|^{1 / 2} \mid \approx 0.044, \\
& \left\{\begin{array}{l}
\left.\theta_{\nu_{\mu} \nu_{\tau}}^{\text {osc }} \approx\left|\sqrt{\frac{m_{\mu}}{m_{\tau}}}\right| \frac{\eta-3 \epsilon}{\eta+3 \epsilon}\right|^{1 / 2}+\sqrt{\frac{m_{\nu_{2}}}{m_{\nu_{3}}}}|\approx| 0.437+(0.378 \pm 0.03) \mid, \\
\text { Thus, } \sin ^{2} 2 \theta_{\nu_{\mu} \nu_{\tau}}^{\text {osc }} \approx 0.99, \quad\left(\text { for } m\left(\nu_{2}\right) / m\left(\nu_{3}\right) \approx 1 / 7\right),
\end{array}\right. \\
& V_{u s} \approx\left|\sqrt{\frac{m_{d}}{m_{s}}}-\sqrt{\frac{m_{u}}{m_{c}}}\right| \approx 0.20 \text {, } \\
& \left|\frac{V_{u b}}{V_{c b}}\right| \approx \sqrt{\frac{m_{u}}{m_{c}}} \approx 0.07 \text {, } \\
& m_{d}(1 \mathrm{GeV}) \approx 8 \mathrm{MeV} \text {, } \\
& \theta_{\nu_{e} \nu_{\mu}}^{\text {osc }} \approx 0.06 \text { (ignoring non-seesaw contributions; see, however, remarks below) }
\end{aligned}
$$

The Majorana masses of the RH neutrinos $\left(N_{i}\right)$ are given by: ${ }^{13}$

$$
\begin{aligned}
& M_{3} \approx M_{R} \approx 10^{15} \mathrm{GeV}(1 / 2-1) \\
& M_{2} \approx\left|y^{2}\right| M_{3} \approx\left(2.5 \times 10^{12} \mathrm{GeV}\right)(1 / 2-1), \\
& M_{1} \approx\left|x-z^{2}\right| M_{3} \sim(1 / 2-2) 10^{-5} M_{3} \sim 10^{10} \mathrm{GeV}(1 / 4-2) .
\end{aligned}
$$

Note that we necessarily have a hierarchical spectrum for the light as well as the heavy neutrinos (see discussions below on $m_{\nu_{1}}$ ). Leaving out the $\nu_{e}-\nu_{\mu}$ oscillation angle for a moment, it seems remarkable that the first seven predictions in Eq. (16) agree with observations, to within 10\%. Particularly intriguing is the (B-L)-dependent group-theoretic correlation between the contribution from the first term in $V_{c b}$ and that in $\theta_{\nu_{\mu} \nu_{\tau}}^{\text {osc }}$, which explains simultaneously why one is small $\left(V_{c b}\right)$ and the other is large $\left(\theta_{\nu_{\mu} \nu_{\tau}}^{\text {osc }}\right)$ [55]. That in turn provides some degree of confidence in the gross structure of the mass-matrices.

As regards $\nu_{e}-\nu_{\mu}$ and $\nu_{e}-\nu_{\tau}$ oscillations, the standard seesaw mechanism would typically lead to rather small angles as in Eq. (16), within the framework presented above [11. It has, however, been noted recently [31] that small intrinsic (non-seesaw) masses $\sim 10^{-3}$ eV of the LH neutrinos can arise quite plausibly through higher dimensional operators of the form [56]: $W_{12} \supset \kappa_{12} \mathbf{1 6}_{1} \mathbf{1 6}_{2} \mathbf{1 6}_{H} \mathbf{1 6}_{H} \mathbf{1 0}_{H} \mathbf{1 0}_{H} / M_{\text {eff }}^{3}$, without involving the standard seesaw mechanism [20]. One can verify that such a term would lead to an intrinsic Majorana mixing mass term of the form $m_{12}^{0} \nu_{L}^{e} \nu_{L}^{\mu}$, with a strength given by $m_{12}^{0} \approx \kappa_{12}\left\langle\mathbf{1 6}_{H}\right\rangle^{2}(175 \mathrm{GeV})^{2} / M_{\text {eff }}^{3} \sim$ $(1.5-6) \times 10^{-3} \mathrm{eV}$, for $\left\langle\mathbf{1 6}_{H}\right\rangle \approx(1-2) M_{\mathrm{GUT}}$ and $\kappa_{12} \sim 1$, if $M_{\mathrm{eff}} \sim M_{\mathrm{GUT}} \approx 2 \times 10^{16} \mathrm{GeV}[57$. Such an intrinsic Majorana $\nu_{e} \nu_{\mu}$ mixing mass $\sim$ few $\times 10^{-3} \mathrm{eV}$, though small compared to $m\left(\nu_{3}\right)$, is still much larger than what one would generically get for the corresponding term from the standard seesaw mechanism [as in Ref. [11]. Now, the diagonal $\left(\nu_{L}^{\mu} \nu_{L}^{\mu}\right)$ mass-term, arising from the standard seesaw mechanism is expected to be $\sim(3-8) \times 10^{-3} \mathrm{eV}$ for a natural value of $|y| \approx 1 / 20-1 / 15$, say [11]. Thus, taking the net values of $m_{22}^{0} \approx 7 \times 10^{-3}$

\footnotetext{
${ }^{12}$ These predictions are based on the fact that the pattern given in Eq. 7 leads to $m_{b}\left(M_{X}\right) \approx m_{\tau}\left(1-8 \epsilon^{2}\right)$. They also reflect the recent trend in the atmospheric and solar neutrino data which suggests $m\left(\nu_{2}\right) / m\left(\nu_{3}\right) \approx$ $1 / 7$.

${ }^{13}$ The range in $M_{3}$ and $M_{2}$ is constrained by the values of $m\left(\nu_{3}\right)$ and $m\left(\nu_{2}\right)$ suggested by the atmospheric and solar neutrino data.
} 
$\mathrm{eV}, m_{12}^{0} \approx 3 \times 10^{-3} \mathrm{eV}$ as above and $m_{11}^{0} \ll 10^{-3} \mathrm{eV}$, which are all plausible, we obtain $m_{\nu_{2}} \approx 7 \times 10^{-3} \mathrm{eV}, m_{\nu_{1}} \sim(1$ to few $) \times 10^{-3} \mathrm{eV}$, so that $\Delta m_{12}^{2} \approx 5 \times 10^{-5} \mathrm{eV}^{2}$ and $\sin ^{2} 2 \theta_{12}^{\text {osc }} \approx 0.6-0.7$. These go well with the LMA MSW solution of the solar neutrino problem.

In summary, the intrinsic non-seesaw contribution to the Majorana masses of the LH neutrinos (neglected in making the predictions of Eq. (16)) can plausibly have the right magnitude for $\nu_{e}-\nu_{\mu}$ mixing so as to lead to the LMA solution within the $G(224) / S O(10)$ framework, without upsetting the successes of the first seven predictions in Eq. (16). [In contrast to the near maximality of the $\nu_{\mu}-\nu_{\tau}$ oscillation angle, however, which emerges as a compelling prediction of the framework [11, the LMA solution, as obtained above, should, be regarded only as a consistent possibility, rather than as a compelling prediction, within this framework.]

It is worth noting at this point that in a theory leading to Majorana masses of the LH neutrinos as above, one would of course expect the neutrinoless double beta decay process (like $n+n \rightarrow p p e^{-} e^{-}$), satisfying $|\Delta L|=2$ and $|\Delta B|=0$, to occur at some level. Search for this process is most important because it directly tests a fundamental conservation law and can shed light on the Majorana nature of the neutrinos, as well as on certain CP violating phases in the neutrino-system (assuming that the process is dominated by neutrinoexchange). The crucial parameter which controls the strength of this process is given by $m_{e e} \equiv\left|\sum_{i} m_{\nu_{i}} U_{e i}^{2}\right|$. With a non-seesaw contribution leading to $m_{\nu_{1}} \sim$ few $\times 10^{-3} \mathrm{eV}$, $m_{\nu_{2}} \approx 7 \times 10^{-3} \mathrm{eV}, \sin ^{2} 2 \theta_{12} \approx 0.6-0.7$, and an expected value for $\sin \theta_{13} \sim m_{13}^{0} / m_{33}^{0} \sim$ $(1-5) \times 10^{-3} \mathrm{eV} /\left(5 \times 10^{-2} \mathrm{eV}\right) \sim(0.02-0.1)$, one would expect $m_{e e} \approx(1-5) \times 10^{-3} \mathrm{eV}$. Such a strength, though compatible with current limits [58], would be accessible if the current sensitivity is improved by about a factor of 50-100. Improving the sensitivity to this level would certainly be most desirable.

I would now like to turn to a discussion of leptogenesis within the $G(224) / S O(10)$ framework for fermion masses and mixings presented above. Before discussing leptogenesis, we need to discuss, however, the origin of $\mathrm{CP}$ violation within the same framework. The discussion so far has ignored, for the sake of simplicity, possible CP violating phases in the parameters $\left(\sigma, \eta, \epsilon, \eta^{\prime}, \epsilon^{\prime}, \zeta_{22}^{u, d}, y, z\right.$, and $\left.x\right)$ of the Dirac and Majorana mass matrices [Eqs. (7), and (10)]. In general, however, these parameters can and generically will have phases [59]. Some combinations of these phases enter into the CKM matrix and define the Wolfenstein parameters $\rho_{W}$ and $\eta_{W}$ [60, which in turn induce CP violation by utilizing the standard model interactions. As observed in Ref. [61, an additional and potentially important source of CP and flavor violations (as in $K^{0} \leftrightarrow \bar{K}^{0}, B_{d, s} \leftrightarrow \bar{B}_{d, s}, b \rightarrow s \bar{s} s$, etc. transitions) arise in the model through supersymmetry [62], involving squark and gluino loops (box and penguin), simply because of the embedding of MSSM within a string-unified $G(224)$ or $S O(10)$-theory near the GUT-scale, and the assumption that primordial SUSY-breaking occurs near the string scale $\left(M_{\text {string }}>M_{\mathrm{GUT}}\right)$ 63. It is shown in 61] that complexification of the parameters $\left(\sigma, \eta, \epsilon, \eta^{\prime}, \epsilon^{\prime}\right.$, etc.), through introduction of phases $\sim 1 / 20-1 / 2$ (say) in them, can still preserve the successes of the predictions as regards fermion masses and neutrino oscillations shown in Eq. (16), as long as one maintains nearly the magnitudes of the real parts of the parameters and especially their relative signs as obtained in Ref. [11] and shown in Eq. (15) 64]. Such a picture is also in accord with the observed features of $\mathrm{CP}$ and flavor violations in $\epsilon_{K}, \Delta m_{B d}$, and asymmetry parameter in $B_{d} \rightarrow J / \Psi+K_{s}$, while 
predicting observable new effects in processes such as $B_{s} \rightarrow \bar{B}_{s}$ and $B_{d} \rightarrow \Phi+K_{s}[61$.

We therefore proceed to discuss leptogenesis concretely within the framework presented above by adopting the Dirac and Majorana fermion mass matrices as shown in Eqs. (77) and (10) and assuming that the parameters appearing in these matrices can have natural phases $\sim 1 / 20-1 / 2$ (say) with either sign up to addition of $\pm \pi$, while their real parts have the relative signs and nearly the magnitudes given in Eq. (16).

\section{Leptogenesis}

Finally, the observed matter-antimatter asymmetry of the universe provides an additional important clue to physics at truly short distances. This issue has taken a new turn since the discovery of the non-perturbative electroweak sphaleron effects [13], which violate $\mathrm{B}+\mathrm{L}$ but conserve B-L. These remain in thermal equilibrium in the temperature range of 200 $\mathrm{GeV}$ to about $10^{12} \mathrm{GeV}$. As a result, they efficiently erase any pre-existing baryon/lepton asymmetry that satisfies $\Delta(\mathrm{B}+\mathrm{L}) \neq 0$, but $\Delta(\mathrm{B}-\mathrm{L})=0$. This is one reason why standard GUT-baryogenesis satisfying $\Delta(\mathrm{B}-\mathrm{L})=0$ (as in minimal $\mathrm{SU}(5)$ ) becomes irrelevant to the observed baryon asymmetry of the universe ${ }^{14}$. On the other hand, purely electroweak baryogenesis based on the sphaleron effects - although a priori an interesting possibility appears to be excluded for the case of the standard model without supersymmetry, and highly constrained as regards the available parameter space for the case of the supersymmetric standard model, owing to LEP lower limit on Higgs mass $\geq 114 \mathrm{GeV}$. As a result, in the presence of electroweak sphalerons, baryogenesis via leptogenesis [12] appears to be an attractive and promising mechanism to generate the observed baryon asymmetry of the universe.

To discuss leptogenesis concretely within the $\mathrm{G}(224) / \mathrm{SO}(10)$ - framework presented above, I follow the discussion of Ref.[22] and first consider the case of thermal leptogenesis. In the context of an inflationary scenario [65, with a plausible reheat temperature $T_{R H} \sim(1$ to few $) \times 10^{9} \mathrm{GeV}$ (say), one can avoid the well known gravitino problem if $m_{3 / 2} \sim(1$ to 2$) \mathrm{TeV}$ [66] and yet produce the lightest heavy neutrino $N_{1}$ efficiently from the thermal bath if $M_{1} \sim(3$ to 5$) \times 10^{9} \mathrm{GeV}$ (say), in accord with Eq. (17) $\left[N_{2}\right.$ and $N_{3}$ are of course too heavy to be produced at $\left.T \sim T_{R H}\right]$. Given lepton number (and B-L) violation occurring through the Majorana mass of $N_{1}$, and $\mathrm{C}$ and $\mathrm{CP}$ violating phases in the Dirac and/or Majorana fermion mass-matrices as mentioned above, the out-of-equilibrium decays of $N_{1}$ (produced from the thermal bath) into $l+H$ and $\bar{l}+\bar{H}$ and into the corresponding SUSY modes $\tilde{l}+\tilde{H}$ and $\overline{\tilde{l}}+\overline{\tilde{H}}$ would produce a B-L violating lepton asymmetry; so also would the decays of $\tilde{N}_{1}$ and $\tilde{\tilde{N}}_{1}$. Part of this asymmetry would of course be washed out due to inverse decays and lepton number violating $2 \leftrightarrow 2$-scatterings. I will assume this commonly adopted mechanism for the so-called thermal leptogenesis (At the end, I will consider an interesting alternative that would involve non-thermal leptogenesis). This mechanism has been extended to incorporate supersymmetry by several authors (see e.g., 67, 68, 69]). The net lepton asymmetry of the universe $\left[Y_{L} \equiv\left(n_{L}-n_{\bar{L}}\right) / s\right]$ arising from decays of $N_{1}$ into $l+H$

\footnotetext{
${ }^{14}$ Standard GUT-baryogenesis involving decays of X and Y gauge bosons (with $M_{X} \sim 10^{16} \mathrm{GeV}$ ) and/or of superheavy Higgs bosons is hard to realize anyway within a plausible inflationary scenario satisfying the gravitino-constraint [see e.g. E. W. Kolb and M. S. Turner, "The Early Universe", Addison-Wesley, 1990].
} 
and $\bar{l}+\bar{H}$ as well as into the corresponding SUSY modes $(\tilde{l}+\tilde{H}$ and $\overline{\tilde{l}}+\overline{\tilde{H}})$ and likewise from $\left(\tilde{N}_{1}, \overline{\tilde{N}}_{1}\right)$-decays [67, 68, 69] is given by:

$$
Y_{L}=\kappa \epsilon_{1}\left(\frac{n_{N_{1}}+n_{\tilde{N}_{1}}+n_{\tilde{N}_{1}}}{s}\right) \approx \kappa \epsilon_{1} / g^{*}
$$

where $\epsilon_{1}$ is the lepton-asymmetry produced per $N_{1}$ (or $\left(\tilde{N}_{1}+\overline{\tilde{N}}_{1}\right)$-pair) decay (see below), and $\kappa$ is the efficiency or damping factor that represents the washout effects mentioned above (thus $\kappa$ incorporates the extent of departure from thermal equilibrium in $N_{1}$-decays; such a departure is needed to realize lepton asymmetry). ${ }^{15}$ The parameter $g^{*} \approx 228$ is the number of light degrees of freedom in MSSM.

The lepton asymmetry $Y_{L}$ is converted to baryon asymmetry, by the sphaleron effects, which is given by:

$$
Y_{B}=\frac{n_{B}-n_{\bar{B}}}{s}=C Y_{L}
$$

where, for MSSM, $C \approx-1 / 3$. Taking into account the interference between the tree and loop-diagrams for the decays of $N_{1} \rightarrow l H$ and $\bar{l} \bar{H}$ (and likewise for $N_{1} \rightarrow \tilde{l} \tilde{H}$ and $\overline{\tilde{l}} \overline{\tilde{H}}$ modes and also for $\tilde{N}_{1}$ and $\overline{\tilde{N}}_{1}$-decays), the CP violating lepton asymmetry parameter in each of the four channels (see e.g., [68] and 69]) is given by

$$
\epsilon_{1}=\frac{1}{8 \pi v^{2}\left(M_{D}^{\dagger} M_{D}\right)_{11}} \sum_{j=2,3} \operatorname{Im}\left[\left(M_{D}^{\dagger} M_{D}\right)_{j 1}\right]^{2} f\left(M_{j}^{2} / M_{1}^{2}\right)
$$

where $M_{D}$ is the Dirac neutrino mass matrix evaluated in a basis in which the Majorana mass matrix of the RH neutrinos $M_{R}^{\nu}$ [see Eq. (10)] is diagonal, $v=(174 \mathrm{GeV}) \sin \beta$ and the function $f \approx-3\left(M_{1} / M_{j}\right)$, for the case of SUSY with $M_{j} \gg M_{1}$.

Including inverse decays as well as $\Delta L \neq 0$-scatterings in the Boltzmann equations, a recent analysis [70] shows that in the relevant parameter-range of interest to us (see below), the efficiency factor (for the SUSY case) is given by [71]:

$$
\kappa \approx\left(0.7 \times 10^{-4}\right)\left(\mathrm{eV} / \tilde{m}_{1}\right)
$$

where $\tilde{m}_{1}$ is an effective mass parameter (related to $K$ [72]), and is given by [73]:

$$
\tilde{m}_{1} \equiv\left(m_{D}^{\dagger} m_{D}\right)_{11} / M_{1}
$$

Eq. (21) should hold to better than $20 \%$ (say), when $\tilde{m}_{1} \gg 5 \times 10^{-4} \mathrm{eV}$ [70] (This applies well to our case, see below).

I proceed to make numerical estimates of the lepton-asymmetry by taking the magnitudes and the relative signs of the real parts of the parameters $\left(\sigma, \eta, \epsilon, \eta^{\prime}, \epsilon^{\prime}\right.$, and $\left.y\right)$ approximately the same as in Eq. (15), but allowing in general for natural phases in them (as in [61]).

\footnotetext{
${ }^{15}$ The efficiency factor mentioned above, is often expressed in terms of the parameter $K \equiv$ $\left[\Gamma\left(N_{1}\right) / 2 H\right]_{T=M_{1}}$ [65]. Assuming initial thermal abundance for $N_{1}, \kappa$ is normalized so that it is 1 if $N_{1}$ 's decay fully out of equilibrium corresponding to $K \ll 1$ (in practise, this actually requires $K<0.1$ ).
} 


\begin{tabular}{|c|c|c|c|}
\hline & \multicolumn{3}{|c|}{$\left|\zeta_{31}-z\right|$} \\
\cline { 2 - 4 } & $(1 / 200)(1 / 3)$ & $(1 / 200)(1 / 4)$ & $(1 / 200)(1 / 5)$ \\
\hline \hline$\tilde{m}_{1}(\mathrm{eV})$ & $0.83 \times 10^{-2}$ & $0.47 \times 10^{-2}$ & $0.30 \times 10^{-2}$ \\
\hline$\kappa$ & $1 / 73$ & $1 / 39$ & $1 / 24$ \\
\hline$Y_{L} / \sin \left(2 \phi_{21}\right)$ & $-11.8 \times 10^{-11}$ & $-22.4 \times 10^{-11}$ & $-36 \times 10^{-11}$ \\
\hline$Y_{B} / \sin \left(2 \phi_{21}\right)$ & $4 \times 10^{-11}$ & $7.5 \times 10^{-11}$ & $12 \times 10^{-11}$ \\
\hline$\phi_{21}$ & $\sim \pi / 4$ & $\sim \pi / 12-\pi / 4$ & $\sim \pi / 18-\pi / 4$ \\
\hline
\end{tabular}

Table 1: Baryon Asymmetry for the Case of Thermal Leptogenesis

In the evaluation of the lepton asymmetry, I allow for small "31" and "13" entries in $M_{\nu}^{D}$, denoted by $\zeta_{31}$ and $\zeta_{13}$ respectively, which are not exhibited in Eq. (77). Following assignment of flavor-charges [53], these are expected to be of order $(1 / 200)(1$ to $1 / 2)$ (say). As such, they have no noticeable effects on fermion masses and mixings discussed above, but they can be relevant to lepton asymmetry.

Using the values of the parameters $\left(\sigma, \epsilon, \epsilon^{\prime}, y\right.$, and $\left.z\right)$ determined from our consideration of fermion masses (see Eq. (15)) and the expected magnitudes of $\zeta_{31}$ and $z$, one obtains the following estimates (see Ref. 22] for details):

$$
\begin{aligned}
\frac{\left(M_{D}^{\dagger} M_{D}\right)_{11}}{\left(\mathcal{M}_{u}^{0}\right)^{2}} & =\left|3 \epsilon^{\prime}-z(\sigma-3 \epsilon)\right|^{2}+\left|\zeta_{31}-z\right|^{2} \\
& \approx 2.5 \times 10^{-5}(1 / 4 \text { to } 1 / 6) \\
\epsilon_{1} & \approx \frac{1}{8 \pi}\left(\frac{\mathcal{M}_{u}^{0}}{v}\right)^{2}|(\sigma+3 \epsilon)-y|^{2} \sin \left(2 \phi_{21}\right)\left(\frac{-3 M_{1}}{M_{2}}\right) \approx-2.0 \times 10^{-6} \sin \left(2 \phi_{21}\right)
\end{aligned}
$$

where, $\phi_{21}=\arg \left[\left(\zeta_{31}-z\right)\left(\sigma^{*}+3 \epsilon^{*}-y^{*}\right)\right]+\left(\phi_{1}-\phi_{2}\right)$. Here $\left(\phi_{1}-\phi_{2}\right)$ is a phase angle that arises from diagonalization of the Majorana mass matrix $M_{R}^{\nu}$ (see [22]). The effective phase $\phi_{21}$ thus depends upon phases in both the Dirac and the Majorana mass matrices. In writing Eq. (24), we have put $\left(\mathcal{M}_{u}^{0} / v\right)^{2} \approx 1 / 2,|\sigma+3 \epsilon-y| \approx 0.13$ (see Eq. (15) and Ref. 64), and for concreteness (for the present case of thermal leptogenesis) $M_{1} \approx 4 \times 10^{9}$ $\mathrm{GeV}$ and $M_{2} \approx 2 \times 10^{12} \mathrm{GeV}$ [see Eq. (17)]. Since $\left|\zeta_{31}\right|$ and $|z|$ are each expected to be of $(1 / 200)(1 t o 1 / 2)$ (say) by flavor symmetry, we have allowed for a possible mild cancellation between their contributions to $\left|\zeta_{31}-z\right|$ by putting $\left|\zeta_{31}-z\right| \approx(1 / 200)(1 / 2-1 / 5)$. The parameter $\tilde{m}_{1}$, given by Eq. (22), turns out to be (approximately) proportional to $\left|\zeta_{31}-z\right|^{2}$ [see Eq. (23)]. It is given by:

$$
\tilde{m}_{1} \approx\left|\zeta_{31}-z\right|^{2}\left(\mathcal{M}_{u}^{0}\right)^{2} / M_{1} \approx\left(1.9 \times 10^{-2} \mathrm{eV}\right)(1 \text { to } 1 / 6)\left(\frac{4 \times 10^{9} \mathrm{GeV}}{M_{1}}\right)
$$

where, as before, we have put $\left|\zeta_{31}-z\right| \approx(1 / 200)(1 / 2$ to $1 / 5)$. The corresponding efficiency factor $\kappa$ [given by Eq. (21)], lepton and baryon-asymmetries $Y_{L}$ and $Y_{B}$ [given by Eqs. (18) and (19)] and the requirement on the phase-parameter $\phi_{21}$ are listed in Table 1 
The constraint on $\phi_{21}$ is obtained from considerations of Big-Bang nucleosynthesis, which requires $3.7 \times 10^{-11} \lesssim\left(Y_{B}\right)_{B B N} \lesssim 9 \times 10^{-11}$ [23]; this is consistent with the CMB data [24], which suggests somewhat higher values of $\left(Y_{B}\right)_{C M B} \approx(7-10) \times 10^{-11}$ (say). We see that the first case $\left|\zeta_{31}-z\right| \approx 1 / 200(1 / 3)$ leads to a baryon asymmetry $Y_{B}$ that is on the low side of the BBN-data, even for a maximal $\sin \left(2 \phi_{21}\right) \approx 1$. The other cases with $\left|\zeta_{31}-z\right| \approx$ $(1 / 200)(1 / 4$ to $1 / 5)$, which are of course perfectly plausible, lead to the desired magnitude of the baryon asymmetry for natural values of the phase parameter $\phi_{21} \sim(\pi / 18$ to $\pi / 4)$. We see that, for the thermal case, the CMB data would suggest somewhat smaller values of $\left|\zeta_{31}-z\right| \sim 10^{-3}$. This constraint would be eliminated for the case of non-thermal leptogenesis.

We next consider briefly the scenario of non-thermal leptogenesis [74, 75]. In this case the inflaton is assumed to decay, following the inflationary epoch, directly into a pair of heavy RH neutrinos (or sneutrinos). These in turn decay into $l+H$ and $\bar{l}+\bar{H}$ as well as into the corresponding SUSY modes, and thereby produce lepton asymmetry, during the process of reheating. It turns out that this scenario goes well with the fermion masspattern of Sec. 2 [in particular see Eq. (17)] and the observed baryon asymmetry, provided $2 M_{2}>m_{\text {infl }}>2 M_{1}$, so that the inflaton decays into $2 N_{1}$ rather than into $2 N_{2}$ (contrast this from the case proposed in Ref. [74]). In this case, the reheating temperature $\left(T_{\mathrm{RH}}\right)$ is found to be much less than $M_{1} \sim 10^{10} \mathrm{GeV}$ (see below); thereby (a) the gravitino constraint is satisfied quite easily, even for a rather low gravitino-mass $\sim 200 \mathrm{GeV}$ (unlike in the thermal case); at the same time (b) while $N_{1}$ 's are produced non-thermally (and copiously) through inflaton decay, they remain out of equilibrium and the wash out process involving inverse decays and $\Delta L \neq 0$-scatterings are ineffective, so that the efficiency factor $\kappa$ is 1 .

To see how the non-thermal case can arise naturally, we recall that the VEV's of the Higgs fields $\Phi=(1,2,4)_{H}$ and $\bar{\Phi}=(1,2, \overline{4})_{H}$ have been utilized to (i) break SU(2) $R$ and B-L so that G(224) breaks to the SM symmetry [5], and simultaneously (ii) to give Majorana masses to the RH neutrinos via the coupling in Eq. (9) (see e.g., Ref. [1]; for $\mathrm{SO}(10), \bar{\Phi}$ and $\Phi$ would be in $\mathbf{1 6}_{H}$ and $\mathbf{1 6} \mathbf{6}_{H}$ respectively). It is attractive to assume that the same $\Phi$ and $\bar{\Phi}$ (in fact their $\nu_{\mathrm{RH}}$ and $\overline{\tilde{\nu}}_{\mathrm{RH}}$-components), which acquire GUT-scale VEV's, also drive inflation [74. In this case the inflaton would naturally couple to a pair of RH neutrinos by the coupling of Eq. (91). To implement hybrid inflation in this context, let us assume following Ref. 74, an effective superpotential $W_{\mathrm{eff}}^{\mathrm{inf}}=\lambda S\left(\bar{\Phi} \Phi-M^{2}\right)+$ (non-ren. terms), where $S$ is a singlet field [76]. It has been shown in Ref. 74 that in this case a flat potential with a radiatively generated slope can arise so as to implement inflation, with $G(224)$ broken during the inflationary epoch to the SM symmetry. The inflaton is made of two complex scalar fields (i.e., $\theta=\left(\delta \tilde{\nu}_{H}^{C}+\delta \tilde{\bar{\nu}}_{H}^{C}\right) / \sqrt{2}$ that represents the fluctuations of the Higgs fields around the SUSY minimum, and the singlet $\mathrm{S})$. Each of these have a mass $m_{\text {infl }}=\sqrt{2} \lambda M$, where $M=\left\langle(1,2,4)_{H}\right\rangle \approx 2 \times 10^{16}$ $\mathrm{GeV}$ and a width $\Gamma_{\mathrm{infl}}=\Gamma\left(\theta \rightarrow \Psi_{\nu_{H}} \Psi_{\nu_{H}}\right)=\Gamma\left(S \rightarrow \tilde{\nu}_{H} \tilde{\nu}_{H}\right) \approx[1 /(8 \pi)]\left(M_{1} / M\right)^{2} m_{\text {infl }}$ so that

$$
T_{\mathrm{RH}} \approx(1 / 7)\left(\Gamma_{\mathrm{infl}} M_{\mathrm{Pl}}\right)^{1 / 2} \approx(1 / 7)\left(M_{1} / M\right)\left[m_{\mathrm{infl}} M_{\mathrm{Pl}} /(8 \pi)\right]^{1 / 2}
$$

For concreteness, take [77] $M_{2} \approx 2 \times 10^{12} \mathrm{GeV}, M_{1} \approx 2 \times 10^{10} \mathrm{GeV}$ (1 to 2) [in accord with Eq. (17)], and $\lambda \approx 10^{-4}$, so that $m_{\text {infl }} \approx 3 \times 10^{12} \mathrm{GeV}$. We then get: $T_{\mathrm{RH}} \approx(1.7 \times$ 
$\left.10^{8} \mathrm{GeV}\right)(1$ to 2$)$, and thus (see e.g., Sec. 8 of Ref. [65]):

$$
\begin{aligned}
\left(Y_{B}\right)_{\text {Non-Thermal }} & \approx-\left(Y_{L} / 3\right) \\
& \approx(-1 / 3)\left[\left(n_{N_{1}}+n_{\tilde{N}_{1}}+n_{\bar{N}_{1}}\right) / s\right] \epsilon_{1} \\
& \approx(-1 / 3)\left[(3 / 2)\left(T_{\mathrm{RH}} / m_{\mathrm{infl}}\right) \epsilon_{1}\right] \\
& \approx\left(30 \times 10^{-11}\right)\left(\sin 2 \phi_{21}\right)(1 \text { to } 2)^{2}
\end{aligned}
$$

Here we have used Eq. (24) for $\epsilon_{1}$ with appropriate $\left(M_{1} / M_{2}\right)$, as above. Setting $M_{1} \approx 2 \times 10^{10}$ for concreteness, we see that $Y_{B}$ obtained above agrees with the (nearly central) observed value of $\left\langle Y_{B}\right\rangle_{\mathrm{BBN}(\mathrm{CMB})}^{\text {central }} \approx(6(8.6)) \times 10^{-11}$, again for a natural value of the phase parameter $\phi_{21} \approx \pi / 30(\pi / 20)$. As mentioned above, one possible advantage of the non-thermal over the thermal case is that the gravitino-constraint can be met rather easily, in the case of the former (because $T_{R H}$ is rather low $\sim 10^{8} \mathrm{GeV}$ ), whereas for the thermal case there is a significant constraint on the lowering of the $T_{R H}$ (so as to satisfy the gravitino-constraint) vis a vis a raising of $M_{1} \sim T_{R H}$ so as to have sufficient baryon asymmetry (note that $\epsilon_{1} \propto M_{1}$, see Eq. (24) ). Furthermore, for the non-thermal case, the dependence of $Y_{B}$ on the parameter $\left|\zeta_{31}-z\right|^{2}$ (which arises through $\kappa$ and $\tilde{m}_{1}$ in the thermal case, see Eqs. (21), (22)), and (23)) is largely eliminated. Thus the expected magnitude of $Y_{B}$ (Eq. (27) holds without a significant constraint on $\left|\zeta_{31}-z\right|$ (in contrast to the thermal case).

To conclude this part, I have considered two alternative scenarios (thermal as well as non-thermal) for inflation and leptogenesis. We see that the $G(224) / S O(10)$ framework provides a simple and unified description of not only fermion masses and neutrino oscillations (consistent with maximal atmospheric and large solar neutrino oscillation angles) but also of baryogenesis via leptogenesis, for the thermal as well as non-thermal cases, in accord with the gravitino constraint. Each of the features - (a) the existence of the right-handed neutrinos, (b) B-L local symmetry, (c) quark-lepton unification through SU(4)-color, (d) the seesaw mechanism, and (e) the magnitude of the supersymmetric unification-scale - plays a crucial role in realizing this unified and successful description. These features in turn point to the relevance of either the $\mathrm{G}(224)$ or the $\mathrm{SO}(10)$ symmetry being effective between the string and the GUT scales, in four dimensions. While the observed magnitude of the baryon asymmetry seems to emerge naturally from within the framework, understanding its observed sign (and thus the relevant CP violating phases) remains a challenging task.

\section{Proton Decay: The Hallmark of Grand Unification}

\subsection{Preliminaries}

Turning to proton decay, I present now the reason why the unification framework based on SUSY SO(10) or SUSY G(224), together with the understanding of fermion masses and mixings discussed above, strongly suggest that proton decay should be imminent.

In supersymmetric unified theories there are in general three distinct mechanisms for proton decay - two realized sometime ago and one rather recently. Briefly, they are:

1. The familiar $d=6$ operators mediated by $\mathrm{X}$ and $\mathrm{Y}$ gauge bosons of $S U(5)$ or $S O(10)$ (Fig. 1). These lead to $e^{+} \pi^{0}$ as the dominant mode. 
2. The "standard" $d=5$ operators [8] (Fig. 2) which arise through exchanges of color triplet Higgsinos which are in the $\mathbf{5}_{\mathrm{H}}+\overline{\mathbf{5}}_{\mathrm{H}}$ of $S U(5)$ or $10_{\mathrm{H}}$ of $S O(10)$. In the presence of these operators, one crucially needs, for consistency with the empirical lower limit on proton lifetime, a suitable doublet-triplet splitting mechanism which assigns GUT-scale masses to the color triplets in the $\mathbf{1 0}_{\mathrm{H}}$ of $S O(10)$ while keeping the electroweak doublets light (see e.g. Ref. [1] for discussion of such mechanisms and relevant references). Following the constraints of Bose symmetry, color antisymmetry and hierarchical Yukawa couplings, these standard $d=5$ operators lead to dominant $\bar{\nu} K^{+}$ and comparable $\bar{\nu} \pi^{+}$modes, but in all cases to highly suppressed $e^{+} \pi^{0}, e^{+} K^{0}$ and even $\mu^{+} K^{0}$ modes.

3. The "new" $d=5$ operators 32 which arise (see Fig. 3) through exchanges of color triplet Higgsinos in the Higgs multiplets like $\mathbf{1 6}_{\mathrm{H}}+\overline{\mathbf{1 6}}_{\mathrm{H}}$ of $S O(10)$, which are used to give superheavy Majorana masses to the RH neutrinos. These operators generically arise through the joint effects of (a) the couplings as in Eq. (9) which assign superheavy Majorana masses to the RH neutrinos, and (b) the couplings of the form $g_{i j} \mathbf{1 6}_{i} \mathbf{1 6}_{j} \mathbf{1 6}_{\mathrm{H}} \mathbf{1 6} \mathbf{H}_{\mathrm{H}} / M$ as in Eq. (8) which are needed to generate CKM mixings (see Sec. 3). Thus these new $d=5$ operators are directly linked not only to the masses and mixings of quarks and leptons, but also to the Majorana masses of the RH neutrinos.

The contributions of these three operators to proton decay has been considered in detail in Ref. [31], which provides an update in this regard of the results of Ref. 11]. Here, I will highlight only the main ingredients that enter into the calculations of proton decay rate, based on the three contributions listed above, and then present a summary of the main results. The reader is referred to these two references for a more detailed presentation and explanations.

Relative to other analyses, the study of proton decay carried out in Refs. [11] and 31] have the following distinctive features:

(i) Link with Fermion Masses: It systematically takes into account the link that exists between the $d=5$ proton decay operators and the masses and mixings of all fermions including neutrinos, within a realistic $G(224) / S O(10)$-framework, as discussed in Sec. 3 .

(ii) Inclusion of the standard and the new $d=5$ operators: In particular, it includes contributions from both the standard and the new $d=5$ operators (Fig. 3), related to the Majorana masses of the RH neutrinos. These latter, invariably omitted in the literature, are found to be generally as important as the standard ones.

(iii) Restricting GUT-scale Threshold Corrections: The study restricts GUTscale threshold corrections to $\alpha_{3}\left(m_{Z}\right)$ so as to be in accord with the demand of "natural" coupling unification. This restriction is especially important for SUSY SO(10), for which, following the mechanism of doublet-triplet splitting (see Appendix of Ref. [11]), the standard $d=5$ operators become inversely proportional to an effective mass-scale given by $M_{\text {eff }} \equiv$ $\left[\lambda\left\langle\mathbf{4 5} 5_{\mathrm{H}}\right\rangle\right]^{2} / M_{10^{\prime}} \sim M_{X}^{2} / M_{10^{\prime}}$, rather than to the physical masses of the color-triplets in the $\mathbf{1 0}_{\mathrm{H}}$ of $S O(10)$. Here $M_{10^{\prime}}$ represents the mass of $\mathbf{1 0}_{\mathrm{H}}^{\prime}$, that enters into the D-T splitting mechanism through an effective coupling $\lambda \mathbf{1 0}_{\mathrm{H}} \mathbf{4 5}_{\mathrm{H}} \mathbf{1 0}_{\mathrm{H}}^{\prime}$ in the superpotential. Now, $M_{10^{\prime}}$ can be naturally suppressed compared to $M_{X}$ owing to flavor symmetries, and thus $M_{\text {eff }}$ can be 
correspondingly larger than $M_{X}$ by even two to three orders of magnitude. ${ }^{16} 17$

Although $M_{\text {eff }}$ can far exceed $M_{X}$, it still gets bounded from above by demanding that coupling unification, as observed, ${ }^{18}$ should emerge as a natural prediction of the theory as opposed to being fortuitous. That in turn requires that there be no large (unpredicted) cancellation between GUT-scale threshold corrections to the gauge couplings that arise from splittings within different multiplets as well as from Planck scale physics. Following this point of view, we have argued (see Ref. [1]) that the net "other" threshold corrections to $\alpha_{3}\left(m_{Z}\right)$ arising from the Higgs and the gauge multiplets should be negative, but conservatively and quite plausibly no more than about $10 \%$, at the electroweak scale. Such a requirement is in fact found to be well satisfied not only in magnitude but also in sign by the minimal Higgs system consisting of $\left(\mathbf{4 5} \mathbf{H}_{\mathrm{H}}, \mathbf{1 6} \mathbf{6}_{\mathrm{H}} \overline{\mathbf{1 6}}_{\mathrm{H}}\right.$, and $\left.\mathbf{1 0} \mathbf{H}_{\mathrm{H}}\right)$ [1]. This in turn restricts how big can be the threshold corrections to $\alpha_{3}\left(m_{Z}\right)$ that arise from (D-T) splitting (which is positive). Since the latter turns out to be proportional to $\ln \left(M_{\text {eff }} \cos \gamma / M_{X}\right)$, we thus obtain an upper limit on $M_{\text {eff }} \cos \gamma$, where $\cos \gamma \approx(\tan \beta) /\left(m_{t} / m_{b}\right)$. An upper limit on $M_{\text {eff }} \cos \gamma$ thus provides an upper limit on $M_{\text {eff }}$ which is inversely proportional to $\tan \beta$. In this way, our demand of natural coupling unification, together with the simplest model of D-T splitting, introduces an upper limit on $M_{\text {eff }}$ given by $M_{\text {eff }} \leq 2.7 \times 10^{18} \mathrm{GeV}(3 / \tan \beta)$ for the case of MSSM embedded in $S O(10)$. This in turn introduces an implicit dependence on $\tan \beta$ into the lower limit of the $\mathrm{SO}(10)$-amplitude -i.e. $\widehat{A}(S O(10)) \propto 1 / M_{\text {eff }} \geq[($ a quantity $) \propto \tan \beta]$. These considerations are reflected in the results given below. [More details can be found in Ref.[11] and [31]].

(iv) Allowing for the ESSM Extension of MSSM: The case of the extended supersymmetric standard model (ESSM), briefly alluded to in Sec. 2, is an interesting variant of MSSM, which can be especially relevant to a host of observable phenomena, including (a) proton decay, (b) possible departure of muon $(g-2)$ from the SM prediction [79], and (c) a lowering of the LEP neutrino-counting from the SM value of 3 [80]. Briefly speaking, ESSM introduces an extra pair of vectorlike families transforming as $\mathbf{1 6}+\overline{\mathbf{1 6}}$ of $S O(10)$, having masses of order $1 \mathrm{TeV}$ [35, 81]. Adding such complete $S O(10)$-multiplets would of course preserve coupling unification. From the point of view of adding extra families, ESSM seems to be the minimal and also the maximal extension of the MSSM, that is allowed in that it is compatible with (a) precision electroweak tests, as well as (b) a semi-perturbative

\footnotetext{
${ }^{16}$ It should be noted that $M_{\text {eff }}$ does not represent the physical masses of the color-triplets or of the other particles in the theory. It is simply a parameter of order $M_{X}^{2} / M_{10^{\prime}}$ that is relevant to proton decay. Thus values of $M_{\text {eff }}$, close to or even exceeding the Planck scale, does not in any way imply large corrections from quantum gravity.

${ }^{17}$ Accompanying the suppression due to $M_{\text {eff }}$, it turns out that the standard $d=5$ operators for $S O(10)$ possess an intrinsic enhancement as well, compared to those for $S U(5)$, primarily due to correlations between the Yukawa couplings in the up and down sectors in $S O(10)$. The standard $d=5$ amplitude for proton decay in $S O(10)$ is thus based on these two opposing effects - suppression through $M_{\text {eff }}$ and enhancement through the Yukawa couplings [11.

${ }^{18}$ For instance, in the absence of GUT-scale threshold corrections, the MSSM value of $\alpha_{3}\left(m_{Z}\right)_{M S S M}$, assuming coupling unification, is given by $\alpha_{3}\left(m_{Z}\right)_{M S S M}^{\circ}=0.125 \pm 0.13$ [10], which is about 5-8\% higher than the observed value: $\alpha_{3}\left(m_{Z}\right)_{M S S M}^{\circ}=0.118 \pm 0.003$. We demand that this discrepancy should be accounted for accurately by a net negative contribution from D-T splitting and from "other" GUT-scale threshold corrections, without involving large cancellations. That in fact does happen for the minimal Higgs system $(\mathbf{4 5}, \mathbf{1 6}, \overline{\mathbf{1 6}})$ (see Ref. [11).
} 
as opposed to non-perturbative gauge coupling unification [35, 82..$^{19}$ The existence of two extra vector-like families of quarks and leptons can of course be tested at the LHC.

Theoretical motivations for the case of ESSM arise on several grounds: (a) it provides a better chance for stabilizing the dilaton by having a semi-perturbative value for $\alpha_{\text {unif }} \approx$ $0.35-0.3$ [35, in contrast to a very weak value of 0.04 for MSSM; (b) owing to increased twoloop effects [35, 82, it raises the unification scale $M_{X}$ to $(1 / 2-2) \times 10^{17} \mathrm{GeV}$ and thereby considerably reduces the problem of a mismatch [36] between the MSSM and the string unification scales (see Section 2); (c) it lowers the GUT-prediction for $\alpha_{3}\left(m_{Z}\right)$ to $(0.112-$ 0.118) (in absence of unification-scale threshold corrections), which is in better agreement with the data than the corresponding value of (0.125-0.113) for MSSM; and (d) it provides a simple reason for inter-family mass-hierarchy [35, 81]. In this sense, ESSM, though less economical than MSSM, offers some distinct advantages.

In the present context, because of raising of $M_{X}$ and lowering of $\alpha_{3}\left(m_{Z}\right)$, ESSM naturally enhances the GUT-prediction for proton lifetime, in full accord with the data [83]. Specifically, for ESSM, one obtains: $M_{\text {eff }} \leq\left(6 \times 10^{18} \mathrm{GeV}\right)(30 / \tan \beta)$ [11, 31].

As a result, in contrast to MSSM, ESSM can allow for larger values of $\tan \beta$ (like 10), or lighter squark masses $(\sim 1 \mathrm{TeV})$ without needing large threshold corrections, and simultaneously without conflicting with the limit on proton lifetime (see below).

\subsection{Proton Decay Rate}

Some of the original references on contributions of the standard $d=5$ operators to proton decay may be found in $[84,85,86,87,88,11,31,89,90]$. I now specify some of the parameters involving the matrix element, renormalization effects and the spectrum of the SUSY partners of the SM particles that are relevant to calculations of proton decay rate.

The hadronic matrix element is defined by $\beta_{H} u_{L}(\vec{k}) \equiv \epsilon_{\alpha \beta \gamma}\left\langle 0\left|\left(d_{L}^{\alpha} u_{L}^{\beta}\right) u_{L}^{\gamma}\right| p, \vec{k}\right\rangle$. A recent improved lattice calculation yields $\beta_{H} \approx 0.014 \mathrm{GeV}^{3}$ [91] (whose systematic errors that may arise from scaling violations and quenching are hard to estimate). We will take as a conservative, but plausible, range for $\beta_{H}$ to be given by $\left(0.014 \mathrm{GeV}^{3}\right)(1 / 2-2)$. $A_{S}$ denotes the short distance renormalization effect for the $d=5$ operator which arises owing to extrapolation between GUT and SUSY-breaking scales [85, 87, 92. The average value of $A_{S}=0.67$, given in Ref. 87] for $m_{t}=100 \mathrm{GeV}$, has been used in most early estimates. For $m_{t}=175 \mathrm{GeV}$, a recent estimate yields: $A_{S} \approx 0.93$ to 1.2 92. Conservatively, I would use $A_{S}=0.93$; this would enhance the rate by a factor of two compared with previous estimates. $A_{L}$ denotes the long-distance renormalization effect of the $d=6$ operator due to QCD interaction that arises due to extrapolation between the SUSY breaking scale and $1 \mathrm{GeV}$ [85. Using the two-loop expression for $A_{L}$ [93], together with the two-loop value of $\alpha_{3}$, Babu and I find: $A_{L} \approx 0.32$. This by itself would also increase the rate by a factor of $(0.32 / 0.22)^{2} \approx 2$, compared to the previous estimates [85, 86, 87, 88, 11. Including the enhancements in both $A_{S}$ and $A_{L}$, we thus see that the net increase in the proton decay rate solely due to new evaluation of renormalization effects is nearly a factor of four, compared to the previous estimates (including that in Ref. [1]).

\footnotetext{
${ }^{19}$ For instance, addition of two pairs of vector-like families at the TeV-scale, to the three chiral families, would cause gauge couplings to become non-perturbative below the unification scale.
} 
In Ref. [1], guided by the demand of naturalness (i.e. the absence of excessive fine tuning), in obtaining the Higgs boson mass, squark masses were assumed to lie in the range of $1 \mathrm{TeV}(1 / \sqrt{2}-\sqrt{2})$, so that $m_{\bar{q}} \lesssim 1.4 \mathrm{TeV}$. Work, based on the notion of focus point supersymmetry however suggests that squarks may be quite a bit heavier without conflicting with the demands of naturalness [94]. In the interest of obtaining a conservative upper limit on proton lifetime, we will therefore allow squark masses to be as heavy as about $2.4 \mathrm{TeV}$.

Allowing for plausible and rather generous uncertainties in the matrix element and the spectrum I take:

$$
\begin{aligned}
\beta_{H} & =\left(0.014 \mathrm{GeV}^{3}\right)(1 / 2-2), \\
m_{\bar{q}} \approx m_{\bar{l}} & \approx 1.2 \mathrm{TeV}(1 / 2-2), \\
m_{\bar{w}} / m_{\bar{q}} & =1 / 6(1 / 2-2), \\
M_{H_{c}}(\text { minimal SU}(5)) & \leq 10^{16} \mathrm{GeV}, \\
A_{L} & \approx 0.32, \\
A_{S} & \approx 0.93 \text { and } \tan \beta \geq 3 .
\end{aligned}
$$

For evaluation of the strengths of the $d=6$ operators, generated by exchanges of $X$ and $Y$ gauge bosons, for the cases of SUSY $S O(10)$ or $S U(5)$ with MSSM spectrum, we take: ${ }^{20}$

$$
\begin{aligned}
& \left.\begin{array}{ccc}
M_{X} \approx M_{Y} & \approx & 10^{16} \mathrm{GeV}(1 \pm 25 \%) \\
\alpha_{i}(G U T) & \approx & 0.04
\end{array}\right\}(\text { for MSSM }) \\
& \alpha_{H}=0.015 \mathrm{GeV}^{3}(1 / 2-2)
\end{aligned}
$$

Before presenting the theoretical predictions, I note the following experimental results on inverse proton decay rates provided by the SuperK studies [95, 83]:

$$
\begin{aligned}
\Gamma^{-1}\left(p \rightarrow e^{+} \pi^{0}\right)_{\operatorname{expt}} & \gtrsim 6 \times 10^{33} \mathrm{yrs} \\
{\left[\sum_{l} \Gamma\left(p \rightarrow \bar{\nu}_{l} K^{+}\right)\right]_{\mathrm{expt}}^{-1} } & \gtrsim 1.9 \times 10^{33} \mathrm{yrs}
\end{aligned}
$$

Before the theoretical predictions for proton decay can be given, a few comments are in order.

1. I present the results separately for the standard $d=5$ and the new $d=5$ operators, allowing for both the MSSM and the ESSM alternatives. (The contributions of the new $d=5$ operators are in fact the same for these two alternatives.) Although the proton decay amplitude receives contributions from both the standard and the new operators, in practice, the standard $d=5$ operators dominate over the new ones for the case of MSSM in the parameter-range of interest that corresponds to predicted proton lifetimes in the upper end, while the new operators dominate over the standard ones for the case of ESSM, in the same range. (This may be inferred from the results listed below.) Thus, in practice, it suffices to consider the contributions of the standard and the new operators separately.

\footnotetext{
${ }^{20}$ For the central value of $\alpha_{H}$, I take the value quoted in Ref. 91] and allow for an uncertainty by a factor of two either way around this central value.
} 
2. In evaluating the contributions of the new $d=5$ operators to proton decay, allowance is made for the fact that for the $f_{i j}$ couplings (see Eq. (9)), there are two possible $S O(10)$ contractions (leading to a $\mathbf{4 5}$ or a $\mathbf{1}$ ) of the pair $\mathbf{1 6} \overline{\mathbf{1 6}}_{H}$, both of which contribute to the Majorana masses of the $\nu_{R} \mathrm{~s}$, but only the contraction via the $\mathbf{4 5}$ contributes to proton decay. In the presence of non-perturbative quantum gravity one would in general expect both contractions to be present having comparable strengths. For example, the couplings of the 45 s lying in the string-tower or possibly below the string scale, and likewise of the singlets, to the $\mathbf{1 6}_{i} \overline{\mathbf{1 6}}_{H}$ pair would respectively generate the two contractions. Allowing for a difference between the relevant projection factors for $\nu_{R^{-}}$ masses versus proton decay operator, we set $\left(f_{i j}\right)_{p} \equiv\left(f_{i j}\right)_{\nu} K$, where $\left(f_{i j}\right)_{\nu}$ defined in Sec. 3 directly yields $\nu_{R}$-masses and $K$ is a relative factor of order unity. ${ }^{21}$ As a plausible range, we take $K \approx 1 / 5-2$ (say), where $K=1 / 5$ seems to be a conservative value on the low side that would correspond to proton lifetimes near the upper end.

The theoretical predictions for proton decay for the case of the minimal supersymmetric SU(5) model, and the supersymmetric SO(10) and G(224)-models developed in Secs. 3 and 4, are summarized below. They are based on (a) the items (i)-(iv) listed above, (b) the two comments mentioned above, and (c) the values of the relevant parameters listed in Eqns. (28) and (29).$^{22}$

\section{A Summary of Results on Proton Decay and Discussions}

$$
\begin{aligned}
& \left.\frac{\text { Min. SUSY } S U(5)}{\operatorname{MSSM}(\text { std. } d=5)}\right\} \Gamma^{-1}\left(p \rightarrow \bar{\nu} K^{+}\right) \leq 1.2 \times 10^{31} \text { yrs } \quad\left(\begin{array}{c}
\text { Excluded by } \\
\text { SuperK }
\end{array}\right) \\
& \left.\frac{\text { SUSY } S O(10)}{\operatorname{MSSM}(\text { std. } d=5)}\right\} \Gamma^{-1}\left(p \rightarrow \bar{\nu} K^{+}\right) \leq 1 \times 10^{33} \text { yrs } \quad\left(\begin{array}{c}
\text { Tightly constrained } \\
\text { by SuperK }
\end{array}\right)
\end{aligned}
$$

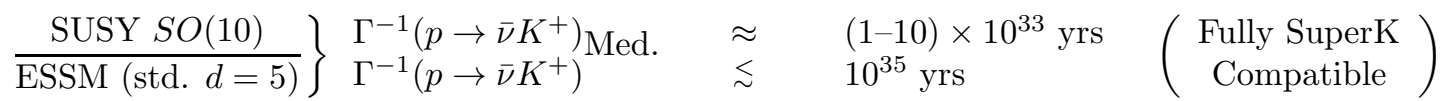

$$
\begin{aligned}
& \left.\frac{\text { SUSY } G(224) / S O(10)}{\text { MSSM or ESSM }(\text { new } d=5)}\right\}\left\{\begin{array}{lll}
\Gamma^{-1}\left(p \rightarrow \bar{\nu} K^{+}\right) \\
B\left(p \rightarrow \mu^{+} K^{0}\right) & \approx & \begin{array}{l}
2 \times 10^{34} \text { yrs } \\
(1-50) \%
\end{array}
\end{array} \quad\left(\begin{array}{c}
\text { Fully Compatible } \\
\text { with SuperK }
\end{array}\right)\right. \\
& \left.\frac{\text { SUSY } S U(5) \text { or } S O(10)}{\operatorname{MSSM}(d=6)}\right\} \Gamma^{-1}\left(p \rightarrow e^{+} \pi^{0}\right) \quad \approx \quad 10^{34.9 \pm 1} \text { yrs } \quad\left(\begin{array}{c}
\text { Fully Compatible } \\
\text { with SuperK }
\end{array}\right)
\end{aligned}
$$

It should be stressed that the upper limits on proton lifetimes given above are quite conservative in that they are obtained (especially for the top two cases) by stretching the uncertainties in the matrix element and the SUSY spectra as given in Eq. (28) to their extremes so as to prolong proton lifetimes. In reality, the lifetimes should be shorter than the upper limits quoted above. With this in mind, the following comments are in order:

\footnotetext{
${ }^{21}$ Thus the new set of proton decay operators become proportional to $\left(f_{i j}\right)_{\nu} g_{k l} K\left\langle\overline{\mathbf{1 6}}_{H}\right\rangle\left\langle\mathbf{1 6}_{H}\right\rangle /\left(M^{2}\right)\left(M_{16}\right)$ where $M \approx M_{\mathrm{st}}$ and $M_{16}\left(\sim M_{\mathrm{GUT}}\right)$ is the mass of the $\mathbf{1 6}_{H}$ (see Ref. 31 for a discussion limiting the strength of this operator).

${ }^{22}$ In obtaining the rate for the $e^{+} \pi^{o}$-mode induced by the $\mathrm{d}=6$ operator, we have used the net renormalization factor $A_{R} \approx 2.5$ representing long and short-distance effects 96 and the chiral lagrangian parameters $-\mathrm{D}$ and $\mathrm{F}$ as in Ref. 97.
} 
1. By comparing the upper limit given in Eq. (311) with the experimental lower limit (Eq. (30)), we see that the minimal SUSY $S U(5)$ with the conventional MSSM spectrum is clearly excluded by a large margin by proton decay searches. This is in full agreement with the conclusion reached by other authors (see e.g. Ref. 90 $)^{23}$. We have of course noted in Sec. 3 that SUSY SU(5) does not go well with neutrino oscillations observed at SuperK.

2. By comparing Eq. (32) with the empirical lower limit (Eq. (301)), we see that the case of MSSM embedded in $S O(10)$ is already tightly constrained to the point of being disfavored by the limit on proton lifetime. The constraint is of course augmented by our requirement of natural coupling unification, which prohibits accidental large cancellation between different threshold corrections. ${ }^{24}$ On the positive side, improvement in the current limit by even a factor of 2-3 (say) ought to reveal proton decay. Otherwise the case of MSSM embedded in $S O(10)$ would be clearly excluded.

3. In contrast to the case of MSSM, that of ESSM embedded in $S O(10)$ (see Eq. (133) ) is fully compatible with the SuperK limit. In this case, $\Gamma_{\text {Med }}^{-1}\left(p \rightarrow \bar{\nu} K^{+}\right) \approx 10^{33}-10^{34}$ yrs, given in Eq. (33), corresponds to the parameters involving the SUSY spectrum and the matrix element $\beta_{H}$ being in the median range, close to their central values (see Eq. (28)). In short, confining to the standard operators only, if ESSM represents low energy physics and if $\tan \beta$ is rather small (3 to 5 say), we do not have to stretch the uncertainties in the SUSY spectrum and the matrix elements to their extreme values in order to understand why proton decay has not been seen as yet, and still can be optimistic that it ought to be discovered in the near future with a lifetime $\lesssim 10^{34} \mathrm{yrs}^{25}$

4. We see from Eq. (34) that the contribution of the new operators related to the Majorana masses of the RH neutrinos (Fig. 3) (which is the same for MSSM and ESSM and is independent of $\tan \beta$ ) is fully compatible with the SuperK limit. These operators can quite naturally lead to proton lifetimes in the range of $10^{33}-10^{34}$ yrs with an upper limit of about $2 \times 10^{34}$ yrs.

It should be remarked that if in the unlikely event all the parameters $\left(\beta_{H}, m_{\tilde{W}} / m_{\tilde{q}}\right.$, and $m_{\tilde{q}}$, etc.) happen to be closer to their extreme values (see Eq. (28) ) so as to extend proton lifetime, the standard $d=5$ operators for the case of ESSM embedded in $S O(10)$ would lead to lifetimes as long as about $10^{35}$ years (see Eq. (33) ). But in this case the new $d=5$ operators related to neutrino masses are likely to dominate and quite naturally lead to lifetimes bounded above in the range of $(1-20) \times 10^{33}$ years (as noted in Eq. (34)). Thus in the presence of the new operators, the range of

\footnotetext{
${ }^{23}$ See, however, Refs 98 and 99, where attempts are made to save minimal SUSY SU(5) by a set of scenarios, which seems (to me) contrived. These include a judicious choice of sfermion mixings, higher dimensional operators and squarks of first two families having masses of order $10 \mathrm{TeV}$.

${ }^{24}$ For instance, had we allowed the "other" GUT-scale threshold corrections (in our case, those arising from $\mathbf{4 5}_{H}, \mathbf{1 6}_{H}, \overline{\mathbf{1 6}}_{H}$ and the gauge multiplet, see Refs. 11] 31]) to $\alpha_{3}\left(m_{Z}\right)$ to be negative in sign and large as about 15\% (rather than 10\%), as some authors do [89, the upper limit on proton lifetime would have been higher by about a factor of 5 , compared to Eq. (32).

${ }^{25}$ The results on proton lifetimes for a wide variation of the parameters for the case of MSSM and ESSM embedded in $S O(10)$ are listed in Tables 1 and 2 of Ref. [31.
} 
$10^{33}-10^{34}$ years for proton lifetime is not only very plausible, but it also provides a reasonable upper limit for the same, for the case of ESSM embedded in SO(10).

5. We see that the gauge boson mediated $d=6$ operators, for the case of MSSM embedded in $S U(5)$ or $S O(10)$, though typically suppressed compared to the $d=5$ operators, can lead to inverse decay rates $\Gamma^{-1}\left(p \rightarrow e^{+} \pi^{0}\right)$ as short as about $10^{34}$ years (see Eq. (35)). It should be stressed that the $e^{+} \pi^{0}$-mode is the common denominator of all GUT models $(S U(5), S O(10)$, etc.) which unify quarks and leptons and the three gauge forces. Its rate is determined essentially by $\alpha_{H}$ and the SUSY unification scale, without the uncertainty of the SUSY spectrum. I should also mention that the $e^{+} \pi^{0}$ mode is predicted to be the dominant mode in the flipped $S U(5) \times U(1)$-model [28], and also as it turns out in certain higher dimensional GUT-models [100, as well as in a model of compactification of M-theory on a manifold of $G_{2}$ holonomy [101]. For these reasons, intensifying the search for the $e^{+} \pi^{0}$-mode to the level of sensitivity of about $10^{35}$ years in a next-generation proton decay detector should be well worth the effort.

It may be noted that for the case of ESSM embedded in $S O(10)$ or $S U(5)$, since $\alpha_{\text {unif }}$ and the unification scale (thereby the masses of the $X, Y$ gauge bosons) are raised by nearly a factor of (6 to 7 ) and (2.5 to 5 ) respectively, compared to those for MSSM (see Ref. 35] and discussions above), while the inverse decay rate is proportional to $\left(M_{X}^{4} / \alpha_{\text {unif }}^{2}\right)$ we expect

$$
\Gamma^{-1}\left(p \rightarrow e^{+} \pi^{0}\right)_{\mathrm{ESSM}}^{d=6} \approx(1 \text { to } 17) \Gamma^{-1}\left(p \rightarrow e^{+} \pi^{0}\right)_{\mathrm{MSSM}}^{d=6} .
$$

This raises the interesting possibility that for ESSM embedded in $S O(10)$, both $\bar{\nu} K^{+}$ (arising from $d=5$ ) and $e^{+} \pi^{0}$ (arising from $d=6$ ) can have comparable rates, with proton having a lifetime $\sim(1 / 2-2) \times 10^{34}$ years.

Before concluding I should mention that there have been several old and new attempts in the literature based on compactification of string/M-theory [101, 102, as well as of a presumed 5D or 6D point-particle GUT-theory [103, 104], which project out the color triplets (anti-triplets) belonging to $\mathbf{5}_{H}\left(\overline{\mathbf{5}}_{H}\right)$ of $S U(5)$ or $\mathbf{1 0}_{H}$ of $S O(10)$ from the massless spectrum in $4 \mathrm{D}$, through the process of compactification. As a result, they obtain a non-GUT SMlike gauge symmetry, and in some cases the $G(224)$ symmetry (see e.g. [17] and [18]) in $4 \mathrm{D}$. In the process they eliminate (often using discrete symmetries) or strongly suppress the standard $d=5$ proton decay operators, though not necessarily the $d=6$.

These approaches are interesting in their own right. There are, however, some constraints which should be satisfied if one wishes to understand certain observed features of low energy physics. In particular, it seems to me that at the very least B-L should emerge as a gauge symmetry in $4 \mathrm{D}$ so as to protect the $\mathrm{RH}$ neutrinos from getting a string-scale mass (see footnote 2) and equally important to implement baryogenesis via leptogenesis, as discussed in Secs. 3 and 4. This feature is not available in models which start with $S U(5)$ in $5 \mathrm{D}$, or in those that obtain only a standard model-like gauge symmetry without B-L in 4D. Furthermore, the full $S U(4)$ color symmetry, which of course contains B-L, plays a crucial role in yielding not only (a) the (empirically favored) relation $m_{b}\left(M_{X}\right) \approx m_{\tau}$, but also (b) the relation $m\left(\nu_{\text {Dirac }}^{\tau}\right)=m_{\text {top }}\left(M_{X}\right)$ which is needed to account for the observed value of 
$\Delta m^{2}\left(\nu_{2}-\nu_{3}\right)$ (see Sec. 3), and (c) the smallness of $V_{c b}$ together with the near maximality of $\sin ^{2} 2 \theta_{\nu_{\mu} \nu_{\tau}}^{\text {osc }}$, as observed. The symmetry $S U(2)_{L} \times S U(2)_{R}$ is also most useful in that it relates the masses and mixings in the up and the down sectors. Without these correlations, the successful predictions listed in Eq. (16) will not emerge.

In short, as noted in Secs. 3 and 4, an understanding of neutrino oscillations and leptogenesis as well as of certain intriguing features of the masses and mixings of all fermions including neutrinos seems to strongly suggest that minimally the symmetry $G(224)$, or maximally the symmetry $S O(10)$, should survive as an effective symmetry in $4 \mathrm{D}$. If the symmetry $G(224)$ rather than $S O(10)$ survives in $4 \mathrm{D}$ near the string scale, the familiar color triplets would be projected out through compactification [see e.g. [17] and [18]. . ${ }^{26}$ In this case, there is no need for a doublet-triplet splitting mechanism and the standard $d=5$ operators are either strongly suppressed or completely eliminated. However, as long as the Majorana masses of the RH neutrinos and the CKM mixings are generated through the minimal Higgs system as in Sec. 3, the new $d=5$ operators (Fig. 3) would still generically be present, and would be the dominant source of proton decay. Like the standard $d=5$ operators (Fig. 2), the new $d=5$ operators also lead to $\bar{\nu} K^{+}$and $\bar{\nu} \pi^{+}$as dominant modes, but in contrast to the standard operators, the new ones can lead to prominent $\mu^{+} K^{0}$-modes [32] (see Eq. (35)).

Given the empirical success of the supersymmetric $G(224) / S O(10)$-framework, derivation of this framework, at least that based on an effective $G(224)$-symmetry in 4D leading to the pattern of Yukawa couplings as in Sec. 3, from an underlying theory remains a challenge. At the same time, based on its empirical support so far, it makes sense to test this picture thoroughly. There are two notable pieces of this picture still missing. One is supersymmetry, which will be tested at the LHC. The other is proton decay.

\subsection{Section Summary}

Given the importance of proton decay, a systematic study of this process has been carried out within a supersymmetric $S O(10) / G(224)$ framework that successfully describes the fermion masses, neutrino oscillations and leptogenesis. Special attention is paid in this study to the dependence of the $\mathrm{d}=5$ proton decay amplitude on the masses and mixings of the fermions and the GUT-scale threshold effects. Allowing for both the MSSM and the ESSM alternatives within this $S O(10) / G(224)$ framework and including the standard as well as the new $d=5$ operators, one obtains (see Eqs. (31)-(35)) a conservative upper limit on proton lifetime given by:

$$
\tau_{\text {proton }} \lesssim(1 / 3-2) \times 10^{34} \text { yrs }\left(\begin{array}{c}
\text { SUSY } \\
S O(10) / G(224)
\end{array}\right)
$$

with $\bar{\nu} K^{+}$and $\bar{\nu} \pi^{+}$being the dominant modes and quite possibly $\mu^{+} K^{0}$ being prominent.

The $e^{+} \pi^{o}$-mode induced by gauge boson-exchanges should have an inverse decay rate in the range of $10^{34}-10^{36}$ years (see Eq. (35)). The implication of these predictions for a next-generation detector is emphasized in the next section.

\footnotetext{
${ }^{26}$ The issue of gauge coupling unification for this case is discussed in Sec. 2.
} 


\section{Concluding Remarks}

In this talk, I have argued that but for two missing pieces - supersymmetry and proton decay - the evidence in favor of supersymmetric grand unification is now strong. It includes: (i) the observed family multiplet-structure, (ii) quantization of electric charge, (iii) the meeting of the three gauge couplings, (iv) neutrino oscillations (atmospheric and solar), (v) the intricate pattern of the masses and mixings of all fermions, including neutrinos, and (vi) the likely need for leptogenesis to account for the observed baryon asymmetry of the universe. All of these features can be understood simply and even quantitatively (see e.g. Eqs. (3), (4), and (16)) within the concept of supersymmetric grand unification based on an effective string-unified $G(224)$ or $S O(10)$-symmetry in 4D. As discussed in Secs 3 and 4, attempts to understand especially (a) the tiny neutrino masses, (b) the baryon asymmetry of the universe (via leptogenesis), as well as (c) certain features of quark-lepton masses and mixings seem to select out the $\mathrm{G}(224) / \mathrm{SO}(10)$ route to unification, as opposed to other alternatives.

A systematic study of proton decay has thus been carried out within this $\mathrm{SO}(10) / \mathrm{G}(224)$ framework [11, 31, allowing for the possibilities of both MSSM and ESSM, and including the contributions for the gauge boson-mediated $d=6$, the standard $d=5$ as well as the new $d=5$ operators related to the Majorana masses of the RH neutrinos. Based on this study, I have

argued that a conservative upper limit on the lifetime of the proton is about $\left(\frac{1}{3}-2\right) \times 10^{34}$ years.

So, unless the fitting of all the pieces (i)-(vi) listed above is a mere coincidence, it is hard to believe that that is the case, discovery of proton decay should be around the corner. Allowing for the possibility that proton lifetime may well be near the upper limit stated above, a next generation detector, of the type proposed by UNO and Hyperkamiokande, providing a net gain in sensitivity by about a factor of five to ten, compared to SuperK, would thus be needed to produce real proton decay events and distinguish them from the background.

The reason for pleading for such improved searches is that proton decay would provide us with a wealth of knowledge about physics at truly short distances $\left(<10^{-30} \mathrm{~cm}\right)$, which cannot be gained by any other means. Specifically, the observation of proton decay, at a rate suggested above, with $\bar{\nu} K^{+}$mode being dominant, would not only reveal the underlying unity of quarks and leptons but also the relevance of supersymmetry. It would also confirm a unification of the fundamental forces at a scale of order $2 \times 10^{16} \mathrm{GeV}$. Furthermore, prominence of the $\mu^{+} K^{0}$ mode, if seen, would have even deeper significance, in that in addition to supporting the three features mentioned above, it would also reveal the link between neutrino masses and proton decay, as discussed in Sec. 5. In this sense, the role of proton decay in probing into physics at the most fundamental level is unique. In view of how valuable such a probe would be and the fact that the predicted upper limit on the proton lifetime is at most a factor of three to ten higher than the empirical lower limit, the argument in favor of building an improved detector seems compelling.

Such a detector should of course be designed to serve multiple goals including especially improved studies of neutrino oscillations and supernova signals. These ideas and others including that of a neutrino factory were discussed intensively at the NeSS meeting held recently in Washington [105].

To conclude, the discovery of proton decay would constitute a landmark in the history of 
physics. That of supersymmetry would do the same. The discoveries of these two features - supersymmetry and proton decay - would fill the two missing pieces of gauge unification and would shed light on how such a unification may be extended to include gravity in the context of a deeper theory. The question thus poses: Will our generation give itself a chance to realize both?

Acknowledgments: I would like to thank Gerard 't Hooft and Antonino Zichichi for the kind invitation to lecture at the Erice School. I have benefitted from many collaborative discussions with Kaladi S. Babu and Frank Wilczek on topics covered in this lecture. I am also grateful to Pasquale Di Bari and Qaisar Shafi for discussions on the topic covered in

Sec. 4. The research presented here is supported in part by DOE grant no. DE-FG02-96ER41015.

\section{References}

[1] Y. Fukuda et al. (Super-Kamiokande), Phys. Rev. Lett. 81, 1562 (1998), hepex/9807003; K. Nishikawa (K2K) Talk at Neutrino 2002, Munich, Germany.

[2] Q. R. Ahmad et al (SNO), Phys. Rev. Lett. 81, 011301 (2002); B. T. Cleveland et al (Homestake), Astrophys. J. 496, 505 (1998); W. Hampel et al. (GALLEX), Phys. Lett. B447, 127, (1999); J. N. Abdurashitov et al (SAGE) (2000), astro-ph/0204245; M. Altmann eta al. (GNO), Phys. Lett. B 490, 16 (2000); S. Fukuda et al. (SuperKamiokande), Phys. Lett. B539, 179 (2002). Disappearance of $\bar{\nu}_{e}$ 's produced in earth-based reactors is established by the KamLAND data: K. Eguchi et al., hepex/0212021. For a recent review including analysis of several authors see, for example, S. Pakvasa and J. W. F. Valle, hep-ph/0301061.

[3] For a historical overview of theoretical calculations of expected solar neutrino flux, see J. Bahcall, astro-ph/0209080.

[4] J. C. Pati, "Implications of the SuperKamiokande Result on the Nature of New Physics", in Neutrino 98, Takayama, Japan, June 98, hep-ph/9807315; Nucl. Phys. B (Proc. Suppl.) 77, 299 (1999).

[5] J. C. Pati and Abdus Salam; Phys. Rev. D8, 1240 (1973); J. C. Pati and Abdus Salam, Phys. Rev. Lett. 31, 661 (1973); Phys. Rev. D10, 275 (1974).

[6] H. Georgi and S. L. Glashow, Phys. Rev. Lett. 32, 438 (1974).

[7] H. Georgi, H. Quinn and S. Weinberg, Phys. Rev. Lett. 33, 451 (1974).

[8] Y. A. Golfand and E. S. Likhtman, JETP Lett. 13, 323 (1971). J. Wess and B. Zumino, Nucl. Phys. B70, 139 (1974); D. Volkov and V. P. Akulov, JETP Lett. 16, 438 (1972).o.

[9] E. Witten, Nucl. Phys. B 188, 513 (1981); R. K. Kaul, Phys. Lett. 109 B, 19 (1982).

[10] The essential features pertaining to coupling unification in SUSY GUTS were noted by S. Dimopoulos, S. Raby and F. Wilczek, Phys. Rev. D 24, 1681 (1981); W. Marciano 
and G. Senjanovic, Phys. Rev. D 25, 3092 (1982) and M. Einhorn and D. R. T. Jones, Nucl. Phys. B 196, 475 (1982). For work in recent years, see P. Langacker and M. Luo, Phys. Rev. D 44, 817 (1991); U. Amaldi, W. de Boer and H. Furtenau, Phys. Rev. Lett. B 260, 131 (1991); F. Anselmo, L. Cifarelli, A. Peterman and A. Zichichi, Nuov. Cim. A 1041817 (1991).

[11] K. S. Babu, J. C. Pati and F. Wilczek, "Fermion Masses, Neutrino Oscillations and Proton Decay in the Light of the SuperKamiokande" hep-ph/981538V3; Nucl. Phys. B (to appear).

[12] M. Fukugita and T. Yanagida, Phys. Lett. B 174, 45 (1986); M. A. Luty, Phys. Rev. D 45, 455 (1992); W. Buchmuller and M. Plumacher, hep-ph/9608308.

[13] V. Kuzmin, V. Rubakov and M. Shaposhnikov, Phys. Lett BM155, 36 (1985).

[14] H. Georgi, in Particles and Fields, Ed. by C. Carlson (AIP, NY, 1975), p. 575; H. Fritzsch and P. Minkowski, Ann. Phys. 93, 193 (1975).

[15] M. Green and J. H. Schwarz, Phys. Lett. 149B, 117 (1984); D. J. Gross, J. A. Harvey, E. Martinec and R. Rohm, Phys. Rev. Lett. 54, 502 (1985); P. Candelas, G. T. Horowitz, A. Strominger and E. Witten, Nucl. Phys. B 258, 46 (1985). For introductions and reviews, see: M. B. Green, J. H. Schwarz and E. Witten, "Superstring Theory" Vols. 1 and 2 (Cambridge University Press); J. Polchinski, "String Theory" vols.1 and 2 (Cambridge University Press).

[16] For a few pioneering papers on string-duality and M-theory, relevant to gauge-coupling unification, see E. Witten, Nucl. Phys. B 443, 85 (1995) and P. Horava and E. Witten, Nucl. Phys. B 460, 506 (1996). For reviews, see e.g. J. Polchinski, hep-th/9511157; and A. Sen, hep-th/9802051, and references therein.

[17] Promising string-theory solutions yielding the G(224)-symmetry in 4D have been obtained using different approaches, by a number of authors. They include: I. Antoniadis, G. Leontaris, and J Rizos, Phys. Lett. B245, 161 (1990); G. K. Leontaris, Phys. Lett. B372, 212 (1996), hep-ph/9601337; A. Murayama and T. Toon, Phys. Lett. B318, 298 (1993); Z. Kakushadze, Phys. rev. D58, 101901 (1998); G. Aldazabal, L. I. Ibanez and F. Quevedo, hep-th/9909172; C. Kokorelis, hep-th/0203187, hep-th/0209202; M. Cvetic, G. Shiu, and A. M. Uranga, Phys. Rev. Lett. 87, 201801 (2001), hep-th/0107143, and Nucl. Phys. B615, 3 (2001), hep-th/0107166; M. Cvetic and I. Papadimitriou, hep-th/0303197; R. Blumenhagen, L. Gorlich and T. Ott, hepth/0211059. For a type I string-motivated scenario leading to the $G(224)$ symmetry in 4D, see L. I. Everett, G. L. Kane, S. F. King, S. Rigolin and L. T. Wang, hepth/0202100.

[18] Recently there have been several attempts based on compactifications of five and sixdimensional GUT-theories which lead to the G(224) symmetry in 4D with certain desirable features. See e.g. R. Dermisek and A. Mafi, Phys. Rev. D65, 055002 (2002) [hep-ph/0108139]; Q. Shafi and Z. Tavartkiladze, hep-ph/0108247, hep-ph/0303150; C. H. Albright and S. M. Barr, hep-ph/0209173; H. D. Kim and S. Raby, hep-ph/0212348; 
I. Gogoladze, Y. Mimura and S. Nandi, hep-ph/0302176; B. Kyae and Q. Shafi, hepph/0211059; H. Baer et al., hep-ph/0204108; For a global phenomenological analysis of a realistic string-inspired supersymmetric model based on the $G(224)$ symmetry see T. Blazek, S. F. King, and J. K. Perry (hep-ph/0303192); and also references therein.

[19] J. C. Pati and Abdus Salam, Phys. Rev. D10, 275 (1974); R. N. Mohapatra and J. C. Pati, Phys. Rev. D11, 566 and 2558 (1975); G. Senjanovic and R. N. Mohapatra, Phys. Rev. D12, 1502 (1975).

[20] M. Gell-Mann, P. Ramond and R. Slansky, in: Supergravity, eds. F. van Nieuwenhuizen and D. Freedman (Amsterdam, North Holland, 1979) p. 315; T. Yanagida, in: Workshop on the Unified Theory and Baryon Number in the Universe, eds. O. Sawada and A. Sugamoto (KEK, Tsukuba) 95 (1979); R. N. Mohapatra and G. Senjanovic, Phys. Rev. Lett. 44, 912 (1980).

[21] F. Gursey, P. Ramond and P. Sikivie, Phys. Lett. B 60, 177 (1976).

[22] J. C. Pati, "Leptogenesis and Neutrino Oscillations Within a predictive G(224)/SO(10) Framework", hep-ph/0209160.

[23] For a recent analysis based on Big Bang Nucleosynthesis, see B. D. Fields and S. Sarkar, Review of Particle Physics, Phys. Rev. D66, 010001 (2002), which yields: $Y_{B}^{B B N} \approx(3.7-9) \times 10^{-11}$.

[24] Most recently, the WMAP surveying the entire celestial sphere with high sensativity yields: $\left(Y_{B}\right)_{\mathrm{WMAP}} \approx(8.7 \pm 0.4) \times 10^{-11}$ (WMAP collaboration, astro-ph/0302207-09$13-15,17,18,20,22-25)$.

[25] M. Dine and A. Kusenko, hep-ph/0303065.

[26] F. Gursey, P. Ramond and R. Slansky, Phys. Lett, B60, 177 (1976); Y. Achiman and B. Stech, Phys. Lett. B77, 389 (1978); Q. Shafi, Phys. Lett. B79, 301 (1978); A. deRujula, H. Georgi and S. L. Glashow, 5th Workshop on Grand Unification, edited by K. Kang et al., World Scientific, 1984, p88.

[27] J. C. Pati and A. Salam, Phys. Rev. D10, 275 (1974); R. N. Mohapatra and J. C. Pati, Phys. Rev. D11, 566 and 2558 (1975).

[28] S. M. Barr, Phys. Lett. B112, 219 (1982); J. P. Derendinger, J. E. Kim and D. V. Nanopoulos, Phys. Lett. B139, 170 (1984); I. Antoniadis, J. Ellis, J. Hagelin and D. V. Nanopoulos, Phys. Lett. B194, 231 (1987).

[29] UNO Proposal: See the talk by C. K. Jung in "Next Generation Nucleon Decay and Neutrino Detector", AIP Conference Proceedings (Sept, 1999), Editors M. Diwan and C. K. Jung.

[30] Plans for Hyperkamiokande: Private Communications, Y. Totsuka. See also talks by M. Shiozawa and Y. Suzuki in the AIP Conf. Proceedings (Ref. [29]). 
[31] J. C. Pati, "Confronting The Conventional ideas on Grand Unification With Fermion Masses, Neutrino Oscillations and Proton Decay", hep-ph/0204240; To appear in the Proceedings of the ICTP, Trieste School and Proceedings of the DAE Meeting, Allahabad, India (2002).

[32] K. S. Babu, J. C. Pati and F. Wilczek, "Suggested New Modes in Supersymmetric Proton Decay", Phys. Lett. B 423, 337 (1998).

[33] P. Ginsparg, Phys. Lett. B 197, 139 (1987); V. S. Kaplunovsky, Nucl. Phys. B 307, 145 (1988); Erratum: ibid. B 382, 436 (1992).

[34] E. Witten, hep-th/9602070.

[35] K. S. Babu and J. C. Pati, "The Problems of Unification - Mismatch and Low $\alpha_{3}$ : A Solution with Light Vector-Like Matter", hep-ph/9606215, Phys. Lett. B 384, 140 (1996).

[36] For a recent discussion, see K. Dienes, Phys. Rep. 287, 447 (1997), hep-th/9602045 and references therein; J. C. Pati, "With Neutrino Masses Revealed, Proton Decay is the Missing Link", hep-ph/9811442; Proc. Salam Memorial Meeting (1998), World Scientific; Int'l Journal of Modern Physics A, vol. 14, 2949 (1999).

[37] See e.g. D. Lewellen, Nucl. Phys. B 337, 61 (1990); A. Font, L. Ibanez and F. Quevedo, Nucl. Phys. B 345, 389 (1990); S. Chaudhari, G. Hockney and J. Lykken, Nucl. Phys. B 456, 89 (1995) and hep-th/9510241; G. Aldazabal, A. Font, L. Ibanez and A. Uranga, Nucl. Phys. B 452, 3 (1995); ibid. B 465, 34 (1996); D. Finnell, Phys. Rev. D 53, 5781 (1996); A.A. Maslikov, I. Naumov and G.G. Volkov, Int. J. Mod. Phys. A 11, 1117 (1996); J. Erler, hep-th/9602032 and G. Cleaver, hep-th/9604183; and Z. Kakushadze and S.H. Tye, hep-th/9605221, and hep-th/9609027; Z. Kakushadze et al., hep-ph/9705202.

[38] A. Faraggi, Phys. Lett. B 278, 131 (1992); Phys. Lett. B 274, 47 (1992); Nucl. Phys. B 403, 101 (1993); A. Faraggi and E. Halyo, Nucl. Phys. B 416, 63 (1994).

[39] A. Faraggi and J.C. Pati, "A Family Universal Anomalous U(1) in String Models as the Origin of Supersymmetry Breaking and Squark-degeneracy", hep-ph/9712516v3, Nucl. Phys. B 256, 526 (1998).

[40] K.S. Babu and J.C. Pati, "A Resolution of Supersymmetric Flavor-changing CP Problems Through String Flavor symmetries", UMD-PP0067, to appear.

[41] J.C. Pati, "The Essential Role of String Derived Symmetries in Ensuring Proton Stability and Light Neutrino Masses", hep-ph/9607446, Phys. Lett. B 388, 532 (1996).

[42] J.C. Pati, (Ref. [36]).

[43] I. Antoniadis, Phys. Lett B246, 377 (1990).

[44] See e.g., J. Lykken, Phys. Rev. D54, 3693 (1996). 
[45] N. Arkani-Hamed, S. Dimopoulos and G. Dvali, Phys. Lett. B429, 263 (1998); I. Antoniadis, N. Arkani-Hamed, S. Dimopoulos and G. Dvali, Phys. Lett. B436, 357 (1998); K. Dienes, E. Dudas, T. Gherghetta, Phys. Lett. B436, 55 (1998). For a recent comprehensive review of this scenario and other references, see N. Arkani-Hamed, S. Dimopoulos and G. Dvali, Physics Today, February 2002 (pages 35-40).

[46] See e.g., K.R. Dienes and J. March-Russell, hep-th/9604112; K.R. Dienes, hep$\mathrm{ph} / 9606467$.

[47] These have been introduced in various forms in the literature. For a sample, see e.g., C. D. Frogatt and H. B. Nielsen, Nucl. Phys. B147, 277 (1979); L. Hall and H. Murayama, Phys. Rev. Lett. 75, 3985 (1995); P. Binetruy, S. Lavignac and P. Ramond, Nucl. Phys. B477, 353 (1996). In the string theory context, see e.g., A. Faraggi, Phys. Lett. B278, 131 (1992).

[48] C. Albright and S. Barr, Phys. Rev. lett. 85, 244 (2000).

[49] For $\mathrm{G}(224)$, one can choose the corresponding sub-multiplets - that is $(1,1,15)_{H},(1$, $2, \overline{4})_{H},(1,2,4)_{H},(2,2,1)_{H}$ - together with a singlet $S$, and write a superpotential analogous to Eq. (8).

[50] If the effective non-renormalizable operator like $\mathbf{1 6}_{2} \mathbf{1 6}_{3} \mathbf{1 0}_{H} \mathbf{4 5}_{H} / M^{\prime}$ is induced through exchange of states with GUT-scale masses involving renormalizable couplings, rather than through quantum gravity, $M^{\prime}$ would, however, be of order GUT-scale. In this case $\left\langle\mathbf{4 5} \mathbf{5}_{H}\right\rangle / M^{\prime} \sim 1$, rather than $1 / 10$.

[51] While $\mathbf{1 6}_{H}$ has a GUT-scale VEV along the SM singlet, it turns out that it can also have a VEV of EW scale along the " $\tilde{\nu}_{L}$ " direction due to its mixing with $\mathbf{1 0}_{H}^{d}$, so that the $H_{d}$ of MSSM is a mixture of $\mathbf{1 0}_{H}^{d}$ and $\mathbf{1 6}_{H}^{d}$. This turns out to be the origin of non-trivial CKM mixings (See Ref. [1]).

[52] The flavor charge(s) of $\mathbf{4 5}_{H}\left(\mathbf{1 6}_{H}\right)$ would get determined depending upon whether $p(q)$ is one or zero (see below).

[53] The basic presumption here is that effective dimensionless couplings allowed by $S O(10) / G(224)$ and flavor symmetries are of order unity [i.e., $\left(h_{i j}, g_{i j}, a_{i j}\right) \approx 1 / 3-3$ (say)]. The need for appropriate powers of $(S / M)$ with $\langle S\rangle / M \sim M_{\mathrm{GUT}} / M_{\mathrm{string}} \sim$ $(1 / 10-1 / 20)$ in the different couplings leads to a hierarchical structure. As an example, consider just one U(1)-flavor symmetry with one singlet S. The hierarchical form of the Yukawa couplings exibited in Eqs. (7) and (8) would be allowed, for the case of $p=1, q=0$, if $\left(\mathbf{1 6}_{3}, \mathbf{1 6}_{2}, \mathbf{1 6}_{1}, \mathbf{1 0}_{H}, \mathbf{1 6}_{H}, \mathbf{4 5}_{H}\right.$ and $\mathrm{S}$ ) are assigned $\mathrm{U}(1)$-charges of ( $a$, $a+1, a+2,-2 a,-a-1 / 2,0,-1)$. It is assumed that other fields are present that would make the U(1) symmetry anomaly-free. With this assignment of charges, one would expect $\left|\zeta_{22}^{u, d}\right| \sim(\langle S\rangle / M)^{2}$; one may thus take $\left|\zeta_{22}^{u, d}\right| \sim(1 / 3) \times 10^{-2}$ without upsetting the success of Ref. [11]. In the same spirit, one would expect $\left|\zeta_{13}, \zeta_{31}\right| \sim(\langle S\rangle / M)^{2} \sim 10^{-2}$ and $\left|\zeta_{11}\right| \sim(\langle S\rangle / M)^{4} \sim 10^{-4}$ (say). The value of "a" would get fixed by the presence of other operators (see later). 
[54] These effective non-renormalizable couplings can of course arise through exchange of (for example) $\mathbf{4 5}$ in the string tower, involving renormalizable $\mathbf{1 6}_{i} \overline{\mathbf{1 6}}_{H} \mathbf{4 5}$ couplings. In this case, one would expect $M \sim M_{\text {string. }}$.

[55] Note that the magnitudes of $\eta, \epsilon$ and $\sigma$ are fixed by the input quark masses. Furthermore, one can argue that the two contributions for $\theta_{\nu_{\mu} \nu_{\tau}}^{\text {osc }}$ [see Eq. (16)] necessarily add to each other as long as $|y|$ is hierarchical $(\sim 1 / 10)[11$. As a result, once the sign of $\epsilon$ relative to $\eta$ and $\sigma$ is chosen to be negative, the actual magnitudes of $V_{c b} \approx(0.044)$ and $\sin ^{2} 2 \theta_{\nu_{\mu} \nu_{\tau}}^{\text {osc }} \approx 0.92-0.99$ emerge as predictions of the model [11, 31].

[56] Note that such an operator would be allowed by the flavor symmetry defined in Ref. 53 if one sets $a=1 / 2$. In this case, operators such as $W_{23}$ and $W_{33}$ that would contribute to $\nu_{L}^{\mu} \nu_{L}^{\tau}$ and $\nu_{L}^{\tau} \nu_{L}^{\tau}$ masses would be suppressed relative to $W_{12}$ by flavor symmetry.

[57] A term like $W_{12}$ can be induced in the presence of, for example, a singlet $\hat{S}$ and a ten-plet (10), possessing effective renormalizable couplings of the form $a_{i} \mathbf{1 6}_{i} \mathbf{1 6}_{H} \hat{\mathbf{1 0}}, b \hat{\mathbf{1 0 1 0}}_{H} \hat{S}$ and mass terms $\hat{M_{S}} \hat{S} \hat{S}$ and $\hat{M_{10}} \hat{\mathbf{1 0 1 0}}$. In this case $\kappa_{12} / M_{\text {eff }}^{3} \approx$ $a_{1} a_{2} b^{2} /\left(\hat{M}_{10}^{2} \hat{M}_{S}\right)$. Setting the charge $a=1 / 2$ (see Ref. [53] and [56]), and assigning charges $(-3 / 2,5 / 2)$ to $(\hat{\mathbf{1 0}}, \hat{S})$, the couplings $a_{1}$, and $b$ would be flavor-symmetry allowed, while $a_{2}$ would be suppressed but so also would be the mass of $\hat{\mathbf{1 0}}$ compared to the GUT-scale. One can imagine that $\hat{S}$ on the other hand acquires a GUT-scale mass through for example the Dine-Seiberg-Witten mechanism, violating the U(1)-flavor symmetry. One can verify that in such a picture, one would obtain $\kappa_{12} / M_{\text {eff }}^{3} \sim 1 / M_{\text {GUT }}^{3}$.

[58] See e.g. P. Vogel, Review of Particle Physics, Phys. Rev. D66, 010001 (2002) for a review of current status and a discussion of the Heidelberg-Moscow collaboration data which suggest a possible signal at the level of 2.2-3.15sigma.

[59] For instance, consider the superpotential for $\mathbf{4 5} \mathbf{5}_{H}$ only: $W\left(\mathbf{4} \mathbf{5}_{H}\right)=M_{45} \mathbf{4 5}{ }_{H}^{2}+\lambda \mathbf{4 5} \mathbf{5}_{H}^{4} / M$, which yields (setting $F_{\mathbf{4 5}_{H}}=0$ ), either $\left\langle\mathbf{4 5}_{H}\right\rangle=0$, or $\left\langle\mathbf{4} \mathbf{5}_{H}\right\rangle^{2}=-\left[2 M_{45} M / \lambda\right]$. Assuming that "other physics" would favor $\left\langle\mathbf{4} \mathbf{5}_{H}\right\rangle \neq 0$, we see that $\left\langle\mathbf{4} \boldsymbol{5}_{H}\right\rangle$ would be pure imaginary, if the square bracket is positive, with all parameters being real. In a coupled system, it is conceivable that $\left\langle\mathbf{4} \mathbf{5}_{H}\right\rangle$ in turn would induce phases (other than "0" and $\pi$ ) in some of the other VEV's as well, and may itself become complex rather than pure imaginary.

[60] L. Wolfenstein. Phys. Rev. Lett., 51, 1983.

[61] K. S. Babu and J. C. Pati. "Link Between Neutrino Oscillations and CP Violation within Supersymmetric Unification". In G. Branco, Q. Shafi, and J. I. Silva-Marcos, editors, Recent Developments in Particle Physics and Cosmology. NATO Advanced Study Institute, June 6-July 7, 2000. Abstract only (paper to appear).

[62] Within the framework developed in Ref. 61, the CP violating phases entering into the SUSY contributions (for example those entering into the squark-mixings), though distinct from the CKM phase, also arise entirely through phases in the fermion mass matrices, just as the CKM phase does. 
[63] An intriguing feature is the prominence of the $\delta_{R R}^{23}\left(\tilde{b}_{R} \rightarrow \tilde{s}_{R}\right)$-parameter which gets enhanced in part because of the largeness of the $\nu_{\mu}-\nu_{\tau}$ oscillation angle. This leads to large departures from the predictions of the standard model, especially in transitions such as $B_{s} \rightarrow \bar{B}_{s}$ and $B_{d} \rightarrow \Phi K_{s}(b \rightarrow s \bar{s} s)$ [61. This feature has independently been noted recently by D. Chang, A. Massiero, and H. Murayama (hep-ph/0205111).

[64] As an example, one such fit with complex parameters assigns [61]: $\sigma=0.10-0.012 i$, $\eta=0.12-0.05 i, \epsilon=-0.095, \eta^{\prime}=4.0 \times 10^{-3}, \epsilon^{\prime}=1.54 \times 10^{-4} e^{i \pi / 4}, \zeta_{22}^{u}=$ $1.25 \times 10^{-3} e^{i \pi / 9}$ and $\zeta_{22}^{d}=4 \times 10^{-3} e^{i \pi / 2}, \mathcal{M}_{u}^{0} \approx 110 \mathrm{GeV}, \mathcal{M}_{D}^{0} \approx 1.5 \mathrm{GeV}$, $y \approx-1 / 17$ (compare with Eq. (15) for which $\zeta_{22}^{u}=\zeta_{22}^{d}=0$ ). One obtains as outputs: $m_{b, s, d} \approx(5 \mathrm{GeV}, 132 \mathrm{MeV}, 8 \mathrm{MeV}), m_{c, u} \approx(1.2 \mathrm{GeV}, 4.9 \mathrm{MeV}), m_{\mu, e} \approx$ $(102 \mathrm{MeV}, 0.4 \mathrm{MeV})$ with $m_{t, \tau} \approx(167 \mathrm{GeV}, 1.777 \mathrm{GeV}), \quad\left(V_{u s}, V_{c b},\left|V_{u b}\right|,\left|V_{t d}\right|\right) \approx$ $(0.217,0.044,0.0029,0.011)$, while preserving the predictions for neutrino masses and oscillations as in Eq. (16). The above serves to demonstrate that complexification of parameters of the sort presented above can preserve the successes of Eq. (16]) (11]). This particular case leads to $\eta_{W}=0.29$ and $\rho_{W}=-0.187$ [61], to be compared with the corresponding standard model values (obtained from $\epsilon_{K}, V_{u b}$ and $\Delta m_{B d}$ ) of $\left(\eta_{W}\right)_{\mathrm{SM}} \approx 0.33$ and $\left(\rho_{W}\right)_{\mathrm{SM}} \approx+0.2$. The consistency of such values for $\eta_{W}$ and $\rho_{W}$ (especially reversal of the sign of $\rho_{W}$ compared to the SM value), in the light of having both standard model and SUSY-contributions to CP and flavor-violations, and their distinguishing tests, are discussed in Ref. 61.

[65] For reviews, see chapters 6 and 8 in E. W. Kolb and M. S. Turner, The Early Universe, Addison-Wesley, 1990.

[66] J. Ellis and J. E. Kim and D. Nanopoulos, Phys. Lett. 145B 181 (1984); M. Yu. Khlopov and A. Linde, Phys. Lett. 138B 265 (1984); E. Holtmann and M. Kawasaki and K. Kohri and T. Moroi, hep-ph/9805405.

[67] B. A. Campbell, S. Davidson and K. A. Olive, Nucl. Phys. B399, 111 (1993).

[68] L. Covi, E. Roulet and F. Vissani, Phys. Lett. B384, 169 (1996).

[69] M. Plumacher, hep-ph/9704231.

[70] P. Di Bari, hep-ph/0211175; W. Buchmuller, P. Di Bari and M. Plumacher, Nucl. Phys. B643, 367 (2002) [hep-ph/0205349].

[71] The factor 0.7 in Eq. (21) [instead of 1 in Eq. (13) of the first paper in Ref. [70] is an estimate that incorporates the modification needed for SUSY corresponding to a doubling of $N_{1}$-decay width owing to the presence of both $l+H$ and $\tilde{l}+\tilde{H}$-modes and an increase of $g^{*}$ from 106 for the standard model to 228 for SUSY.

[72] One can verify that $K \equiv\left(\Gamma\left(N_{1}\right) / 2 H\right)_{T=M_{1}} \approx(0.37)\left[M_{\mathrm{Pl}} /\left(1.66 \sqrt{g^{*}}\left(8 \pi v^{2}\right)\right)\right] \tilde{m}_{1} \approx$ $234\left(\tilde{m}_{1} / \mathrm{eV}\right)$, where 0.37 denotes the usual time-dilation factor, $g^{*}($ for SUSY $) \approx 230$ and $v \approx 174 \mathrm{GeV}$. For comparison, we note that if one includes only inverse decays (thus neglecting $\Delta L \neq 0$-scatterings) in the Boltzmann equations, one would obtain: $\kappa \approx 0.3 /\left[K(\ln K)^{0.6}\right]$ for $K>10$ [65, and $\kappa \approx 1 / 2 K$ for $1 \lesssim K \lesssim 10$. As pointed 
out in Ref. [70], these expressions, frequently used in the literature, however, tend to overestimate $\kappa$ by nearly a factor of 7 . In what follows, we will therefore use Eq. (21) to evaluate $\kappa$.

[73] M. Plumacher, Z. Phys. C74, 549 (1997).

[74] For a specific scenario of inflation and leptogenesis in the context of SUSY G(224), see R. Jeannerot, S. Khalil, G. Lazarides and Q. Shafi, JHEP 010, 012 (2000) (hepph/0002151), and references therein. As noted in this paper, with the VEV's of $(1,2$, $4)_{H}$ and $(1,2, \overline{4})_{H}$ breaking $\mathrm{G}(224)$ to the standard model, and also driving inflation, just the COBE measurement of $\delta T / T \approx 6.6 \times 10^{-6}$, interestingly enough, implies that the relevant $\mathrm{VEV}$ should be of order $10^{16} \mathrm{GeV}$. In this case, the inflaton made of two complex scalar fields (i.e., $\theta=\left(\delta \tilde{\nu}_{H}^{c}+\delta \tilde{\bar{\nu}}_{H}^{c}\right) / \sqrt{2}$, given by the fluctuations of the Higgs fields, and a singlet $S$ ), each with a mass $\sim 10^{12}-10^{13} \mathrm{GeV}$, would decay directly into a pair of heavy RH neutrinos - that is into $N_{2} N_{2}$ (or $N_{1} N_{1}$ ) if $m_{\text {infl }}>2 M_{2}$ (or $2 M_{1}$ ). The subsequent decays of $N_{2}$ 's (or $N_{1}$ 's), thus produced, into $l+\Phi_{H}$ and $\bar{l}+\bar{\Phi}_{H}$ would produce lepton-asymmetry during the process of reheating. I will comment later on the consistency of this possibility with the fermion mass-pattern exhibited in Sec. 2. I would like to thank Qaisar Shafi for a discussion on these issues.

[75] K. Kumekawa, T. Moroi and T. Yanagida, Prog. Theor. Phys. 92, 437 (1994); G. F. Giudice, M. Peleso, A. Riotto and T. Tkachev, JHEP 9908, 014 (1999) [hepph/9905242]; T. Asaka, K. Hamaguchi, M. Kawasaki and T. Yanagida, Phys. Lett. B464, 12 (1999) [hep-ph/9906366].

[76] Incorporating such an effective superpotential in accord with the assignment of flavorcharges suggested in Refs. [53] and [57] would involve two additional singlets with appropriate charges. The $(\mathrm{VEV})^{2}$ of one or both of these may represent $M^{2}$. Derivation of such a picture with appropriate flavor-charge assignments from an underlying (string/M) theory still remains a challenge.

[77] Note that for this non-thermal case, since the gravitino-constraint is relaxed, $N_{1}$ can be chosen heavier than for the case considered before (the thermal case), still in accord with Eq. (17). Since $Y_{B} \propto \epsilon_{1} T_{\mathrm{RH}} / m_{\text {infl }}$, while $\epsilon_{1} \propto\left(M_{1} / M_{2}\right), T_{\mathrm{RH}} \propto M_{1}\left(m_{\mathrm{infl}}\right)^{1 / 2}$ and $m_{\text {infl }} \propto \lambda$, we see that $Y_{B} \propto\left(M_{1}^{2} / M_{2}\right) / \sqrt{\lambda}$, for a constant $M$, for the case of non-thermal leptogenesis.

[78] N. Sakai and T. Yanagida, Nucl. Phys. B 197, 533 (1982); S. Weinberg, Phys. Rev. D 26, 287 (1982).

[79] K. S. Babu and J. C. Pati, "Radiative Processes $\left(\tau \rightarrow \mu \gamma, \mu \rightarrow e \gamma\right.$, and $\left.(g-2)_{\mu}\right)$ as Probes of ESSM/SO(10)", hep-ph/0207289.

[80] K. S. Babu and J. C. Pati, "Neutrino Counting, NuTeV Measurements, Higgs Mass and $V_{u s}$ as Probes of Vectorlike families in ESSM/SO(10)", hep-ph/0203029.

[81] J.C. Pati, Phys. Lett. B228, 228 (1989); K.S. Babu, J.C. Pati and H. Stremnitzer, Phys. Rev. D 51, 2451 (1995); K.S. Babu, J.C. Pati and X. Zhang, Phys. Rev. D46, 21990 (1992). 
[82] C. Kolda and J. March-Russell, Phys. Rev. D 554252 (1997); R. Hempfing, Phys. Lett 351, 206 (1995); M. Bastero-Gil and B. Brahmachari, Nuc. Phys. B575, 35, (2000).

[83] SuperK Collaboration: Y. Hayato, Proc. ICHEP, Vancouver (1998); M. Earl, NNN2000 Workshop, Irvine, Calif (Feb, 2000); Y. Totsuka (private comm. May, 2001); M. Vagins, Report on SuperK Results presented at WHEPP-7 meeting, Allahabad, India (January 6, 2002).

[84] S. Dimopoulos, S. Raby and F. Wilczek, Phys. Lett. B112, 133 (1982).

[85] J. Ellis, D.V. Nanopoulos and S. Rudaz, Nucl. Phys. B 202, 43 (1982).

[86] P. Nath, A.H. Chemseddine and R. Arnowitt, Phys. Rev. D 32, 2348 (1985); P. Nath and R. Arnowitt, hep-ph/9708469.

[87] J. Hisano, H. Murayama and T. Yanagida, Nucl. Phys. B 402, 46 (1993). For a recent estimate of the lifetime for the $d=6$ gauge boson mediated $e^{+} \pi^{0}$-mode, see J. Hisano, hep-ph/0004266.

[88] K.S. Babu and S.M. Barr, Phys. Rev. D 50, 3529 (1994); D 51, 2463 (1995).

[89] V. Lucas and S. Raby, Phys. Rev. D55, 6986 (1997); R. Darmisek, A. Mafi and S. Raby, hep-ph/0007213, V2.

[90] H. Murayama and A. Pierce, hep-ph/0108104.

[91] S. Aoki et al., JLQCD collaboration, hep-latt/9911026; Phys. Rev. D 62, 014506 (2000).

[92] K. Turznyski, hep-ph/0110282, V2.

[93] J. Arafune and T. Nihei, Prog. Theor. Phys. 93, 665.

[94] J.L. Feng, K.T. Matchev and T. Moroi, Phys. Rev. D 61, 75005 (2000), hep$\mathrm{ph} / 9909334$.

[95] SuperK bound on $\Gamma^{-1}\left(p \rightarrow e^{+} \pi^{o}\right)$; See e.g. Ref. [83].

[96] L. I. Ibanez and C. Munoz, Nucl. Phys. B245, 425 (1984).

[97] M. Claudson, M. B. Wise and L. J. Hall, Nucl. Phys. B195, 297 (1982); S. Chadha and M. Daniels, Nucl. Phys. B229, 105 (1983); S. J. Brodsky, J. Ellis, J. S. Hagelin and C. Sachrajda, Nucl. Phys. B238, 561 (1984).

[98] B. Bajc, P. Fileviez Perez and G. Senjanovic, hep-ph/0204311 and hep-ph/0210374.

[99] D. Emmanuel-Costa and S. Wiesenfeldt, hep-ph/0302272.

[100] See e.g. L. Hall and Y. Nomura, hep-ph/0103125; hep-ph/0205067. These papers provide examples of higher dimensional GUTs which lead to prominent $\mathrm{d}=6$ proton decay via the $e^{+} \pi^{o}$ mode. Alternative cases lead to dominance of the $\mu^{+} K^{o}$-mode: See e.g. Y. Nomura, hep-ph/0108070; A. Hebecker and J. March-Russell, hep-ph/0204037. 
[101] T. Friedman and E. Witten (hep-th/0211269).

[102] See e.g. Candelas et al in Ref. [15]; Ref. [17] and [38].

[103] For attempts of this type leading to standard model-like gauge symmetry in 4D, see e.g. Y. Kawamura, Prog. Theor. Phys. 105, 999 (2001); hep-ph/0012125; L.J. Hall and Y. Nomura, Phys. Rev. D64, 055003, hep-ph/0103125; G. Altarelli and F. Feruglio, Phys. Lett. B511, 257 (2001); hep-ph/0102301; A. B. Kobakhidze, hep-ph/0102323; M. Kakizaki and M. Yamaguchi, hep-ph/0104103; A. Hebecker and J. March-Russell, hepph/0107039; hep-ph/0204037; L.J. Hall and Y. Nomura, hep-ph/0111068; T. Asaka and W. Buchmuller and L. Covi, Phys. Lett., B523 (2001); L.J. Hall, Y. Nomura, T. Okui and D. Smith, hep-ph/0108071.

[104] For attempts based on higher dimensional GUTs leading to the G(224) symmetry in 4D, see Ref. 18. For a short discussion of such attempts and relevant references, see Sec. 6.7 of Ref. 31].

[105] International Workshop on Neutrino and Subterranean Science (NeSS 2002 Meeting), Washington, D.C., September (2002). For transparencies of various talks see http://www.physics.umd.edu/NeSS02. 


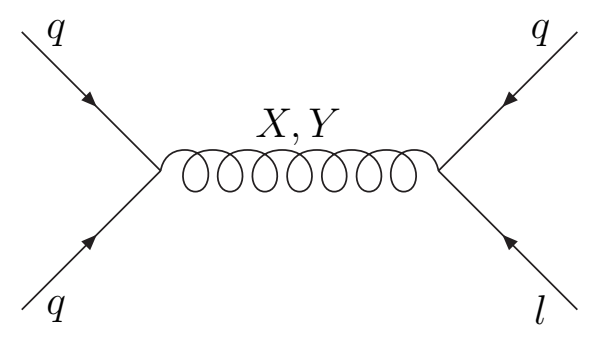

Figure 1: $d=6$ proton decay operator.

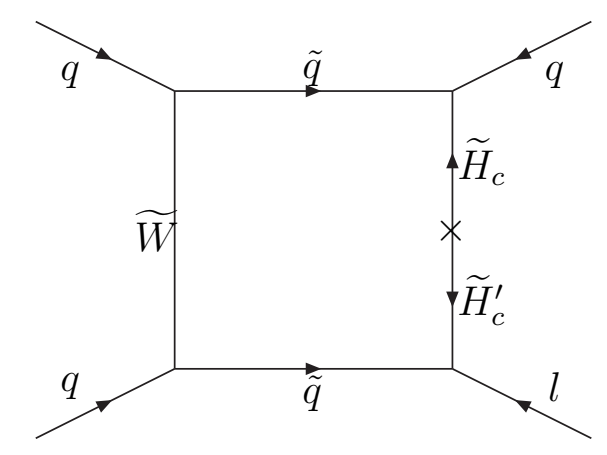

Figure 2: The standard $d=5$ proton decay operator. The $\widetilde{H}_{c}^{\prime}\left(\widetilde{H}_{c}\right)$ are color triplet(antitriplet) Higgsinos belonging to $5_{H}\left(\overline{5}_{H}\right)$ of $S U(5)$ or $10_{H}$ of $S O(10)$.

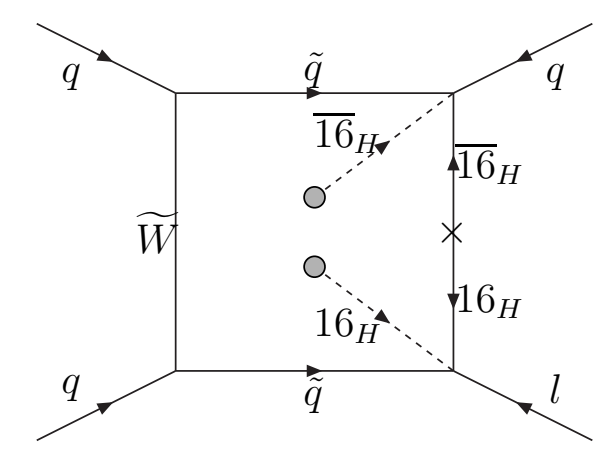

Figure 3: The "new" $d=5$ operators related to the Majorana masses of the RH neutrinos. Note that the vertex at the upper right utilizes the coupling in Eq.(9) which assigns Majorana masses to $\nu_{R}$ 's, while the lower right vertex utilizes the $g_{i j}$ couplings in Eq.(8) which are needed to generate CKM mixings. 